\title{
A!
}

This is an electronic reprint of the original article.

This reprint may differ from the original in pagination and typographic detail.

Ali-Vehmas, Timo

\section{Complex network perspective on collaboration in the ICT standardization}

\section{Published in:}

International Journal of Standardization Research

DOI:

10.4018/IJSR.2016070103

Published: 01/07/2016

Document Version

Publisher's PDF, also known as Version of record

Please cite the original version:

Ali-Vehmas, T. (2016). Complex network perspective on collaboration in the ICT standardization. International Journal of Standardization Research, 14(2), 33-64. https://doi.org/10.4018/lJSR.2016070103

This material is protected by copyright and other intellectual property rights, and duplication or sale of all or part of any of the repository collections is not permitted, except that material may be duplicated by you for your research use or educational purposes in electronic or print form. You must obtain permission for any other use. Electronic or print copies may not be offered, whether for sale or otherwise to anyone who is not an authorised user. 


\title{
Complex Network Perspective on Collaboration in the ICT Standardization
}

Timo Ali-Vehmas, Aalto University, Communications and Networking, Espoo, Finland

\begin{abstract}
Standardization is a crucial enabler of global business of Information and Communications Technologies. Convergence of the underlying networking paradigms of licensed Mobile Communication and license exempted Internet has made progress but full integration is still far from being complete. For standardization professionals the unpredictable convergence makes decisionmaking and participation in standardization complicated. This study examines collaboration in five closely related standardization organizations working in this field during the years from 2003 to 2008 . The results show similarities and differences in collaboration structures and behaviours reflecting the specific scope and context of each standardization organization. Furthermore, this study extends the use of social network analysis as a tool to the field of empirical standardization research. The results pave the way to better collaboration in standardization communities of converging Mobile Internet and beyond by providing better visibility and new insights to standardization leaders, policy makers and users.
\end{abstract}

\section{KEYWORDS}

Collaboration, Internet, Mobile Communications, Networks, Social Network Analysis, Standardization

\section{INTRODUCTION}

Standardization (Swann, 2010) and Information and Communications Technologies (ICT) standardization specifically (Shin, Kim, \& Hwang, 2015) have been studied extensively. Standards and standardization are major drivers of choice and change. Standards are known to enable ecosystems where complementary products utilize open interfaces (Katz \& Shapiro, 1985). Emerging network effects reflect the strength and type of ties defined by standardized interfaces. Network effects motivate companies to voluntarily contribute their proprietary technologies to open standardization (Economides, 1996) while too strong network effects create undesired technology lock-in as is the case in the QWERTY keyboard (David, 1985). High expectations related to the network effects can make incompatible competition more lucrative for leading dominant companies. Therefore, open interoperability through standardization must be favoured by public policy makers (Farrell \& Klemperer, 2007).

Linkages between standardization and business models have increased. Scope of standardization has expanded to new areas when focused collaborative consortia have emerged to address weaknesses of the traditional formal standardization (Hawkins \& Ballon, 2007), (Blind \& Gauch, 2008). Number and volume of different standardization activities has spawned as needs for interoperability, compatibility, scale of economies and for faster innovation diffusion have increased in the globalized markets (Choi, Kim, \& Lee, 2010; Rogers, 1995).

Extensive use of the ICT technologies spreads even broader in our society today when the $5 \mathrm{G}$, Internet of Things and consumers' data driven applications are emerging. The ICT standardization 
with multiple parallel processes needs more clarity and better structures. Traditional classification of standardization leans to the question whether an activity has a formal legal status defined by regulation (de jure) or if market actors drive the effort (de facto). The de jure standards may be promulgated directly by governmental agencies (mandated) or be based on a collaborative work in standards writing organizations (committee) having a formal delegated ("licensed") position. Long time ago governmental organizations alone took care of standards for telecommunications. This approach has almost disappeared except in few areas like national security. Authorized organizations such as the European Telecommunications Standards Institute ${ }^{1}$ (ETSI) create most of the formal standards for telecommunications today.

De facto standards may have a dedicated sponsor or owner, which have interest and full control over the standard (proprietary platform leader controlling the publicly available interface specifications) or an "unsponsored" standard is an outcome of a voluntary open collaboration of interested actors. This last model is the most rapidly growing area of standardization, the Bluetooth ${ }^{2}$ community being one example. The key difference between the two de facto standardization approaches is the level of openness and control of the standard and the standardization process (David \& Greenstein, 1990; Funk \& Methe, 2001; Gandal, Salant, \& Waverman, 2003). As a summary, Table 1 shows a simplified categorization.

This study examines collaborative standards setting within five key Standards Setting Organizations $^{3}$ (SSO) of Mobile Communications and Internet. The $3{ }^{\text {rd }}$ Generation Partnership Project (3GPP) is authorized to develop specifications for licensed network operators providing public mobile communications service. The Open Mobile Alliance (OMA) was set up to coordinate the development of service enablers for mobile communications. The Internet Engineering Task Force (IETF) has assumed responsibility to develop protocols for the Internet while the focus of the World Wide Web Consortium (W3C) is on standards for web technologies. The Local Area Network (LAN) standards committee for wireless LAN (802.11) in the Institute of Electrical and Electronics Engineers (IEEE) focuses on standards for technologies also known as Wi-Fi. These five forums form the critical core of relevant standardization for global ICT market. Furthermore, the critical interplay of the SSOs address challenges and uncertainties of the multifaceted transition of the traditional mainstream cellular telephony to wireless Internet or alternatively issues in making the Internet mobile (Tilson \& Lyytinen, 2006). The focus period of this research is covering the years 2003 -2008 when the Mobile Internet convergence took its first major steps.

Social network analysis (SNA) of a large data set derived from documents contributed in these five SSOs provides the quantitative baseline while qualitative observations based on selected expert interviews build bridges between the SNA results and practical work in the standardization. One additional aim is to promote SNA methodology as a tool for standardization research. This study aims to focus on networking and peaceful collaboration rather than fierce competition.

The overall purpose of this research is to reduce uncertainties related to standardization. Standards developers and users want to gain better portfolio decisions (Toppila, Liesiö, \& Salo, 2011) and define more successful product plans and platform road maps (Gaynor \& Bradner, 2001). Forum shopping is an approach for technology providers (Lerner \& Tirole, 2006). Commercial product implementers, however, face different and more severe challenge when integrated product designs

Table 1. Simplified categorization of the system archetypes (ecosystem dynamics) of different compatibility seeking approached (adapted from (Ali-Vehmas \& Casey, 2012))

\begin{tabular}{|l|l|l|}
\hline & \multicolumn{1}{|c|}{ Mandated/Non-Collaborative } & \multicolumn{1}{c|}{ Collaborative } \\
\hline Public de jure & 1: Mandated (by government) & 2: Delegated to authorized actors \\
\hline Private de facto & 4: Proprietary dominant design & 3. Voluntary collaboration \\
\hline
\end{tabular}


have to support multiple competing standards in parallel and when the decision-making and leadership of standardization collaboration is a complex multi-forum dilemma.

Chapter 2 defines the research framework based on earlier literature and chapter 3 describes the research area covering the five selected SSOs. Chapter 4 introduces the research methodology and empirical data while chapter 5 presents the main findings of the quantitative analysis. Chapter 6 aims to qualitatively discuss and evaluate the findings. Finally, chapter 7 is a short set of conclusions. The appendix 1 provides a brief theoretical background of SNA with definitions of the SNA terminology used in this research.

\section{RESEARCH FRAMEWORK}

Standardization has been considered as a competition between different technologies, different business models and between different SSOs. Success of a standardization process depends on a large number of different factors including characteristics of the supporting companies, standardized technology itself and actions of all the stakeholders (Kaa van de \& Vries, 2015) as well as on network effects and life cycle dynamics of the standard (Blind, 2011). New needs for research arise related to question on how standardization entities develop over time. When originally small and agile market driven standardization organization gains recognition and develops its processes the initial clear scope may become ambiguous (Pohlmann, 2014). Growing number of market driven standardization consortia parallel to the progressing convergence of the ICT with all other sectors of life have created a need to look at the competition and collaboration of the standardization groups as a larger network of standards and standardization (Jakobs, 2003; Jakobs, 2008; Baron, Meniere, \& Pohlmann, 2014). Collaboration networks in standardization do not emerge randomly but a number of factors characterises successful and less successful coalitions. Alliance formation is a strategic not a statistical game (Garas, Tomasello, \& Schweitzer, 2014).

Competition and collaboration between companies as well as between SSOs create an invisible fabric of complex dependencies. Flexibility of standard (Ende van den, Kaa van de, Uijl den, \& Vries de, 2012) and characteristics of collaborator network (Kaa van de, Ende van den, Vries de, \& Heck van, 2011) have been recognized to improve likelihood of success. Distributional conflicts in standards are difficult to agree (Simcoe, 2012). However, high ratio of the same companies participating in both the related industry consortia and the main standardization project significantly correlates with the success of those companies promoting their technical ideas and solutions in the main standardization project. Like-mindedness of the member companies and their delegates develops in those consortia which helps them to learn from each other both technical and business aspects needed in the standardization (Leiponen, 2008). Like-mindedness is also an organizational element. Inter-organizational learning involving corporatist firms significantly increases when the dominant logic within the arena is also corporatist. When a pluralist logic dominates the arena, corporatist dyads (company pairs where both companies have corporatist background) learn less because firms in the dyad activate a contradictory logic that decouples them from their natural processes for inter-organizational learning (Vasudeva, Alexander, \& Jones, 2015).

Standardization is not only a technical but also highly social process. "While standards might aim at the creation of stability and sameness, standardization itself is a highly dynamic phenomenon. Even the stability of standards themselves has to be understood as the result of underlying dynamic processes" (Brunsson, Rasche, \& Seidl, 2012). The multiple dynamical relationships are either competitive, cooperative or simultaneously both, i.e. coopetitive ${ }^{4}$ (Majchrzak, Jarvenpaa, \& Bagherzadeh, 2015). There is a strong causality between standards, organizational relationships around the standards and the diffusion of the services based on the standards in wireless technologies (Lyytinen \& King, 2002). Literature is developing to study broader collaboration aspects of standards setting including information exchange and development of complementing assets (Bar \& Leiponen, 
2014) and innovations (Delcamp \& Leiponen, 2014). Complexity of the collaborative networks is a growing challenge for the future standardized technologies (Katusic, Weber, Bojic et al., 2012).

For complex problems, general systems theory is a source of inspiration (von Bertalanffy, 1972). and concerns (Sterman, 2002) and systems thinking is a mechanism to address the "big things" related also to standardization (Swann, 2010). Research on complex systems utilizes methodologies developed for graph and network theories. Formal quantitative methods such as social network analysis (SNA) open up specific new perspectives on complexities of collaborative networks. Social networks consist of participating collaborators (network node, vertex) and the artefacts of the collaboration (network links, edges). SNA tools provide insightful information on the collaboration beyond simple metrics like the number of standardization contributions per actor. SNA explores relationships between collaborating nodes assessed by e.g. different centrality measures (Wasserman \& Faust, 1994). The relational ties between nodes are channels for transfer of resources and knowledge and therefore the networks provide both opportunities and constraints for individual actors. In order to be more central a node has to be close to all other nodes but additionally the node has to be connected to other wellconnected nodes in the network (Hanneman \& Riddle, 2005). SNA tools have been applied e.g. to business, medicine and computing, for instance to network of hyperlinks on web pages pointing to other web pages of the World Wide Web (Barabási \& Bonabeau, 2003).

Social networks typically consist of few nodes, which are highly connected and a long tail of less connected nodes. The node and link distributions of many social networks follow the so-called power law. Power law distributions have been observed also in new business models where products and services are produced by crowds in a decentralized manner and where consumers have become prosumers (Anderson, 2006). Scarcity of a key resource efficiently limits emergence of such a crowd by letting only few players to participate. This is likely to affect the network node distribution also. Furthermore, the mechanism how new nodes are connected to the existing network, i.e. preferential attachment, influences strongly the network dynamics. For instance, in so-called scale free networks the preference is linearly depending on the number of existing nodes and links in the networks. If the preference linkage is stronger the network grows faster and it is likely to experience the "Winner take all" scenario and eventually the network assumes a star topology with one central hub (Barabási \& Bonabeau, 2003). In case of regional innovation clusters, hierarchical and assortative characteristics of the collaboration networks indicate their capability to cope with radical changes in their environmental conditions (Crespo, Suire, \& Vicente, 2014). The SNA research on innovation networks of mobile phone industry in Europe have shown similar results (Crespo, Suire, \& Vicente, 2016). When analysing very large data sets, like software contributions in open source software projects such as FLOSS community (Free, Libre, Open Source Software) the SNA tools become necessity. The different measures of centrality provide deep insights to the collaboration within the networks including development over time (Martinez-Romo, Robles, Gonzalez-Barahona et al., 2008).

Recently SNA has been utilized also to study different types of consortia in the mobile standardsetting to identify for instance the controlling parties in a collaboration network in China (Kwak, Lee, \& Chung, 2012). Similarly, SNA tools have been used to analyse the changes of the interdependency of the RFCs ${ }^{5}$ as a measure of collaboration and clustering in IETF (Gençer, 2007). This study focuses on the co-authorship networks as suggested in (Gençer, 2012) and aims to provide a further analysis on collaboration in the five key SSOs of Mobile Communication and Internet.

\section{STANDARDIZATION OF INFORMATION AND COMMUNICATIONS TECHNOLOGIES}

The 3GPP Release 99 was the first set of specifications for Mobile Communications with full global scope. During the $3 \mathrm{G}$ era, the market became ready for a serious debate on digital convergence. New requirements of the consumers brought the Internet technologies into the Mobile Communications and at the same time, separate ambitions to make the Internet mobile started to grow. Current integrated 
multi-mode mobile devices include many standardized functionalities such as $2 \mathrm{G}, 3 \mathrm{G}$ and $4 \mathrm{G}$ cellular radio network technologies with Subscriber Identity Module (SIM) based identity as well as local connectivity technologies such as IEEE $802.11 \mathrm{a} / \mathrm{b} / \mathrm{g} / \mathrm{n} /$. Convergence is strongly visible in the vast range of Internet technologies and World Wide Web (WWW) capabilities as well as in a large amount of vendor specific and platform driven software functionalities and applications running on the mobile devices today. Significant part of the standards commercial products utilise today is a result of collaboration, conceptualization and development effort which took place in the five SSOs during the research period of this study. The different layers of the ICT driven business have different drivers and characteristics (Fransman, 2010). Convergence is a different challenge to each SSO due to their different scope in the overall, layered ICT business domain.

The origin of the standardization groups of this study can be traced back to four different types of genesis: governmental organizations (DARPA ${ }^{6}$ for IETF, CERN ${ }^{7}$ for W3C), licensed organizations (ETSI and other similar for $3 \mathrm{GPP}$ ), combination of commercial and voluntary organizations (OMA) and a voluntary society of professional engineers (IEEE ${ }^{8}$ for IEEE 802.11).

The work that later was organized under IETF and $\mathrm{W} 3 \mathrm{C}$ had been initiated by governmental organizations. The Defence Advanced Research Project Agency (DARPA) research played a central role in launching the conceptual work, which led to the Internet while the European organization for Nuclear Research (CERN) project ENQUIRE was the first formal step towards World Wide Web. In both cases, the mission was handed over to a number of leading universities such as Massachusetts Institute of Technology (MIT), University of California Los Angeles (UCLA) and Stanford Research Institute (SRI). Evidently, the formal collaborative organizations IETF and W3C were founded to take over the management responsibilities while the technology contributions by a large number of universities continued actively. The role of universities is visible in the collaboration within these SSOs still during the research period of this study (about 10-15\% of the data points). The development paths have some historical difference also. IETF emerged among several possible technology initiatives (Campbell-Kelly \& Garcia-Swartz, 2013) whereas W3C has a little shorter and less fragmented history of fermentation.

A turning point in the history of IETF took place in 1992 when the Internet Society (ISOC) as a parental legal organization for IETF was established ${ }^{9}$. The process was complete by 1996 . The change in the governance enabled strong growth of the contributor base including many international contributors. Simultaneously the number of internet users experienced a hockey stick growth curve change in $1997^{10}$. Today IETF is the only relevant group developing network protocols for the Internet, most notably the Internet Protocol (IPv4/IPv6) and the Transport Control Protocol (TCP) (Crocker, 1993; Leiner et al., 2009). IETF does not have any specific holistic technical architectural structure but rather the community develops the protocols using a scale free approach in order to support the autonomous re-routing of the data packets. IETF provides communication network layers on the top of the physical link layers, e.g. the functionalities defined by the IEEE 802 group and provides the platform for the content layers defined e.g. in W3C. W3C develops web standards, most notably the Hypertext Markup Language (HTML5). The W3C standards define the Open Web platform, which enables developers to create rich interactive web experiences. Multiple browser implementations can exercise the protocols and utilize the Application Programming Interfaces (API) of the mobile devices allowing the web services and web applications to run on almost any hardware. IETF and later W3C have defined the foundations of web architecture including Hypertext transport protocol (HTTP) and Unified Resource Locators (URL). Close co-operation and structural alignment between IETF and W3C has secured high compatibility and interoperability of the protocols developed by these two SSOs (Simcoe, 2015). The first meeting of the W3 Consortium took place in $1995^{11}$.

When setting up the Internet standardization the initial IETF and W3C development environment were not only formally "liberalized" but rather the communities were simply transferred to a new environment with full, academic freedom. New organizations built their formal structures gradually. 
They did not redesign the rules of governmental organizations but rather removed the old rules completely and let the new rules to emerge bottom-up.

IEEE 802.11 has its foundation in a voluntary collaboration of professional engineers in their social organization, IEEE and its Standards Association (IEEE-SA). IEEE has a remarkable history of over 100 years and an international position with members in about 100 countries. The IEEE-SA membership is twofold, consisting of about 180 corporate members ${ }^{12}$ and the large membership base of individual engineers. The IEEE 802.11 standardization is open for all IEEE members to follow and to participate in the decision-making.

After the liberalization of the specific radio spectrum for Industrial, Scientific and Medical (ISM) use in 1985 IEEE-SA noted a need for a voluntary standard for local area wireless connectivity using this spectrum. Multiple different technologies without any interoperability initially fragmented the field severely. IEEE 802.11 completed its first standard by 1997. This standard utilised earlier technologies, developed in IEEE 802 as the design framework. One key success factor was the networking compatibility with IEEE 802.3 (wired Ethernet). The IEEE 802.11 standards implement the wireless version of the wired Ethernet (Lemstra \& Hayes, 2009).

Furthermore, the purpose of the Wi-Fi Alliance ${ }^{13}$ (WFA) is to increase the market acceptance of the IEEE 802.11 standards. WFA operates as a post processor prioritizing the IEEE 802.11 standards by creating interoperability certifications for selected subsets of the standards. IEEE-SA and therefore, IEEE 802.11 is a recognized standardization organization by ANSI ${ }^{14}$ while the WFA currently is not ${ }^{15}$.

The roots of $3 \mathrm{GPP}$ are deep in the history of telecommunications standardization. A direct continuum from the $2^{\text {nd }}$ generation mobile systems including their promoters, like mobile operators, their product and technology providers as well as the formal standardization organizations in all key markets, Europe, the USA, Japan, China and Korea is evident (Hillebrand, 2013). Related to GSM system evolution the roots of the working processes can be traced back to even further to $1^{\text {st }}$ generation systems (Haug, 2002). The critical border conditions i.e. operators' license requirements and especially fundamentals of radio spectrum regulations have not changed since the 1990's. For instance, the European Conference of Postal and Telecommunications Administrations ${ }^{16}$ (CEPT) still maintains the coordination within Europe. $3 \mathrm{GPP}$ has responded to selected regional differences such as different spectrum bands, emergency call numbers and data security settings by providing fully interoperable, adaptive configurations for each region.

The 3GPP membership is open but in practice requires solid financial resources. All the delegates in the meetings represent their affiliations. In order to become a formal standard, the specifications, created in 3GPP require formal approvals by the Organizational Partners, i.e. the regional and national standardization organizations such as ETSI, ATIS $^{17}$ and CCSA $^{18}$.

3GPP covers functionalities on all layers (Fransman, 2010) while the other standardization groups studied in this research cover smaller areas. 3GPP is a system level standardization organization intending to define a large modular architecture with several open internal and external interfaces (Hillebrand, 2013). A specific System Architecture (SA) group takes care of the conceptual development and other dedicated groups develop the necessary interface standards for modules. This approach creates high-level alignment between technical and organization models. 3GPP also collaborates in some key areas with its organizational partners such as for SIM/USIM card specifications with ETSI (ETSI/SCP). The effort on content and application layer is relatively weak in 3GPP. Home location register (HLR/HSS) represents the identity management of the content layer.

The companies working in the 3GPP ecosystem initiated OMA (Grøtnes, 2008). They noted a need to expand the set of contributors and to include the leading Information Technology (IT) companies into the mobile communication ecosystem in order to build stronger content layer functionalities. The need for OMA emerged because the IT companies did not feel comfortable to join directly the $3 \mathrm{GPP}$ standardization. The IT companies considered the discussions in 3GPP being telecommunications oriented only ${ }^{19}$. Before OMA, these needs were addressed by a number of small separate consortia of like-minded contributors from IT and Communications industries. The momentum however, was 
weak and the consolidation intended to improve the situation. Separate industry initiatives of WAP Forum, Wireless Village, SyncML, Mobile Games and Location Interoperability Forum and Mobile Wireless Internet Forum evidently merged into one single organization.

OMA covers networking applications and specifically standards for underlying service enablers. In the early days, 3GPP and OMA agreed to transfer some application layer functionalities like Multimedia Messaging from 3GPP to OMA. The architectural design in OMA leans towards the 3GPP architecture. Several but not all OMA service enabler protocols are reflections of similar protocols developed in IETF and W3C for the Internet. Conceptually, OMA operates on the top of the 3GPP functionalities and enhances the mobile network capabilities beyond the basic voice and short message services. The OMA membership is available for supporting companies only.

The combined effort of IETF, IEEE 802.11, W3C and other related organizations like the SIP Forum ${ }^{20}$ provides a set of standards for the Internet, which forms a comparable set of the combined functionalities of the OMA and 3GPP standards for the Mobile Communications. Originally, these two set of standards were developed and deployed totally separately. The digital convergence has started to push the systems together forcing the related standardization groups to talk to each other, too. Especially the collaboration between IETF and 3GPP has become important for the support of Internet over Mobile Communications. The Internet Multimedia Subsystem (IMS) of 3GPP is a deliberate action and a major undertaking to seek collaboration between these two SSOs. Furthermore, loosely coupled interoperability has been achieved between the licensed and unlicensed radio technologies (Gunasekaran \& Harmantzis, 2008). However, the projected tightly coupled multimode products utilising Wi-Fi and WiMAX ${ }^{21}$ with $2 \mathrm{G} / 3 \mathrm{G} / 4 \mathrm{G}$ cellular network technologies have not diffused broadly to the market. Figure 1 summarizes historical developments towards a multi-mode mobile device.

Regulatory environment implicitly defines dynamics in competition and collaboration as well as possibilities to build high-level multi-mode functionalities. It is useful to note the difference when the interplay takes place between systems of similar environments like between GSM and CDMA (Cabral \& Salant, 2014) or between different types of setups like between 3GPP and Wi-Fi (Lemstra \& Hayes, 2009; Trestian, Ormond, \& Muntean, 2012). Majority of the earlier research on standardization has been studying aspects of competition between different technologies and standardization organizations.

Figure 1. Convergence of standards in a mobile device (for ecosystem dynamics ref to Table 1)

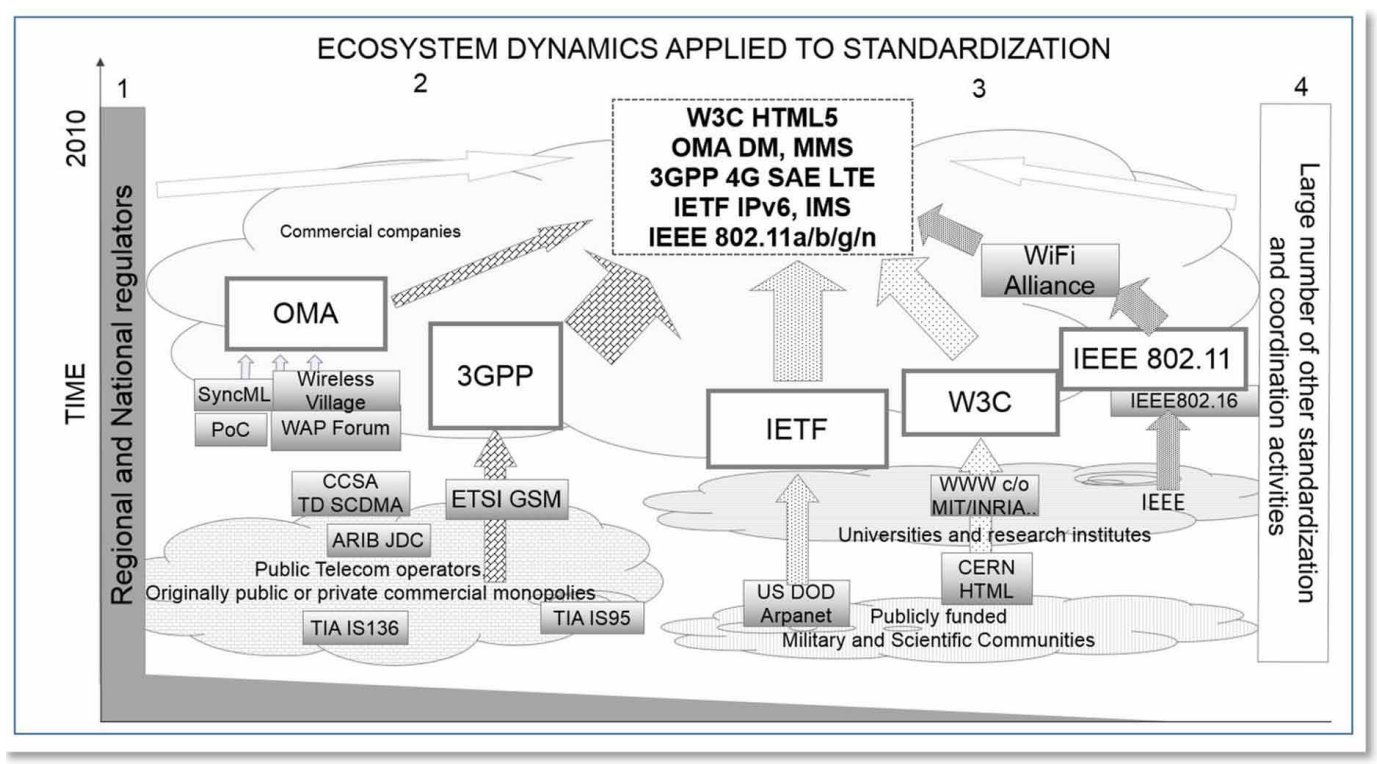


As a complementing step, this research aims to study and compare collaboration in standardization forums based on their internal characteristics. The broader research on collaboration between the standardization organizations is for the future study.

\section{EMPIRICAL RESEARCH METHODOLOGY AND THE DATA}

Main approach of this study is quantitative based on social network analysis. However, limited qualitative elements gained through selected expert interviews are included to complement and to provided deeper insights (Di Minin \& Bianchi, 2011). The challenge while using SNA is to interpret the numerical or graphical results beyond the theoretical models. The benefits of SNA however include the possibility to cover large data sets and condense the information into a low number of specific knowledge points or questions. Personal observations, opinions and preferences, on the other hand approach these questions with deep tacit information. The two approaches are usually only partially valid but by combining the objective results of the SNA with subjective observations of the interviewees there is a possibility to achieve less subjective conclusions and at the same time open up new, more specific research paths for the future. The research focuses on the period of the initial phase of the digital convergence between 2003 and 2008.

\section{Quantitative Research}

Collaboration in writing is an action with an intention to make an impact. Written contributions are considered a meaningful proxy to observe actions of standardization participants (Weiss \& Sirbu, 1990). Furthermore, written document with author's affiliation information typically represent the best interests of the affiliation due to the SSO membership bylaws, processes and traditions as noted in previous studies (Jakobs, Procter, \& Williams, 2001; Isaak, 2006).

There is however, an obvious challenge in modelling standardization collaboration based only on the written documents. A stand-alone contribution may be collaborative in its content. Furthermore, numerous corridor discussions even without any resulting documents are essential in consensus building. Similarly, some single contributions may over time become very valuable, even iconic while the role of other documents is simply to record the process. Still, gaining a public position in standardization between companies in writing always require significant amount of preparations and collaboration and therefore in this research only the written documents are considered.

The empirical data collected from the document repositories of these five forums consist of document headers including the names of the contributors with time stamp. All documents provided by working group leaders, formal editors or chairpersons and similar are omitted because these documents are considered neutral related to collaboration and competition. In case when a SSO contributes to some other SSO (liaison statement), contribution by the SSO is included and affiliated to the contributing SSO itself. Furthermore, "W3C has also its own technical staff able to develop the technology forward" explains a W3C executive. This dual role as a standardization and research organization makes $\mathrm{W} 3 \mathrm{C}$ itself to show up as an active contributor with high number of collaborative contributions. The final set of contributors consists of member organizations of these SSOs (companies, universities, research organizations, governmental actors and standardization organizations themselves) as well as the similar liaising entities. Some joint ventures, mergers and acquisitions between the contributors took place during the research period, which made manual editing necessary on case-by-case bases.

The final data set forms a bipartite network between the contributors and their contributions. The bipartite networks are converted to simpler projections assuming the contributors are connected nodes in a network if they have collaborated to contribute a document while the joint contributions of multiple contributors form the links of the network. Stand-alone contributions do not add any link to the network of collaboration. Therefore, contributors providing only stand-alone contributions form 
a set of disconnected nodes. Similar simplification is used in many earlier social network studies, for instance about collaboration of movie actors (Watts \& Strogatz, 1998) and scientists (Newman, 2001).

Comparable data is available on all selected SSOs from 2003 to 2008. Data outside of this period, however, is used selectively. The sampling rate of the data is one calendar year. Table 2 shows the number of documents used in this analysis as well as the number of the contributing organization.

The interviews note the key role of the leader in the contribution process. Therefore, each document with the names of the contributors forms a small elementary start network as an evidence of collaboration. The lead contributor is the central node in the elementary network and the collaborating contributors connect to the lead contributor over identical undirected links. The elementary star networks combined result the final undirected but weighted network of collaboration. Link weights indicate the total number of joint contributions between the nodes. Weighted and unweighted networks provide two different perspectives on the collaboration. The weights of the weights, i.e. the specific value of each document are not used ${ }^{22}$.

The collaboration network of each SSO forms an adjacency matrix $\mathrm{A}^{23}$. The matrix A has $\mathrm{N}$ columns and $\mathrm{N}$ rows representing the $\mathrm{N}$ contributors in the SSO where the elements $\mathrm{a}_{\mathrm{ij}}$ show the number of collaborative contributions between the contributors i and j. For detailed analysis, a separate adjacency matrix is created for each SSO for each year. Time series of the adjacency matrices enable flexible way to study development of the collaboration over a research period.

\section{Qualitative Research}

The participating people create the standards. Interviews of 13 highly experienced standardization leaders and contributors provide basis for the qualitative findings. All the interviewed experts have a minimum of 5 years of experience in hands-on standardization work in several different standardization organization including at least one of the SSOs studied in this paper. They represent different roles including company lead delegate, document editor, chairperson of a sub group or of a technical plenary, chair or a member of the board of a SSO as well as director of a SSO with an overall operative or legal or some other executive level responsibility. The qualitative data collection took place between April 2015 and March 2016 in face-to-face and virtual semi-structured interviews. The interviews addressed the whole lifespan of the SSOs with the special focus on the years of the quantitative data (2003 - 2008).

\section{RESULTS OF THE QUANTITATIVE EMPIRICAL RESEARCH}

Standardization is a contribution driven process where the contributions are focused in the early phase on new work items with conceptual and architectural requirements while towards the end of the process more detailed comments and change requests dominate (Leiponen, 2008). Many standardization organizations are running several parallel processes and therefore the mixture of contributions in a standards meeting include different types of documents and statements.

Table 2. Number of contributors and their contributions in the SSOs. The comparable data is available for the years $2003-$ 2008.

\begin{tabular}{|l|c|c|c|c|c|}
\hline & OMA & 3GPP & IETF & W3C & IEEE 802.11 \\
\hline Contributions (total) & 68066 & 308967 & 55292 & 1243 & 25761 \\
\hline Contributors (total) & 254 & 623 & 2879 & 324 & 402 \\
\hline Contributions 2003-2008 & 65959 & 185789 & 29728 & 846 & 17498 \\
\hline Contributors 2003-2008 & 248 & 415 & 1768 & 267 & 294 \\
\hline
\end{tabular}




\section{Competition, Cooperation, and Coopetition}

In this initial analysis, standardization contributors fall into three groups. One group of contributors consists of Cooperative actors who never contribute alone. They are considered high collaborative (Collaboration $=1$ ), while another group consists of Competitive contributors who never contribute with anybody else (Collaboration $=0$ ). The third group consists of Coopetitive contributors who do both $(0<$ Collaboration $<1)$ (Majchrzak et al., 2015). With this categorization, Table 3 shows the numbers of different contributors in each SSO.

The differences between the shapes of distributions shown in Figure 2 reveal more details. The distribution of different types of contributors forms a collaboration profile for each SSO. The number of contributions each contributor has done is not taken into account in these curves i.e. collaboration profile is unweighted. The total number of all contributors $\left(\mathrm{N}_{\mathrm{SSO}}\right.$ is naturally different for each SSO. Horizontal axis represents all the contributors ranging from 1 to $\mathrm{N}_{\mathrm{Sso}}$ for each $\mathrm{SSO}$, in other words the axis is scaled to fit the contributors into the same range $[0,100 \%]$ Vertical axis shows the ratio in contributions for each contributor within the range $[0,1]$ i.e. $[0,100 \%]$ collaborative.

Starting from the middle, the shapes of the curves are almost identical for OMA and 3GPP where majority of the contributors (70\% for both) is coopetitive. In IEEE 802.11 , collaborative only contributors form a small minority (1.7\%) while majority (59\%) of the contributors are working only alone. W3C is at the opposite mode where half $(51 \%)$ of the companies are collaborative only. The shape of the histogram for IETF is similar to W3C with balanced collaborating and competing contributors being the majority (59\%).

\section{It is a Small World}

The earlier research on "Small World" and random networks has yielded very basic level observation. The ratio of hierarchy (transitivity, clustering) and communications capabilities (path length) of the network forms a simple starting point for the SNA giving an indication of network topology. Neighbours are neighbours even if only one link connects them together. In other words in this section, weight value of all non-zero link equals 1 .

Transitivity in all five SSOs is high when compared to random networks of similar size while the average path length is short. This is an indication of the "Small World" network topology in all SSOs. Transitivity, however in these networks has a trend while the average path length is rather similar for all (see Figure 3). The OMA and 3GPP collaboration networks show higher transitivity and lowest average path length. This indicates higher level of hierarchy of the collaboration in those organizations compared to the other three SSOs. Global transitivity and average path length show similarities between IETF, W3C and IEEE 802.11. This is in particular interesting because the absolute numbers of contributors in IETF, W3C and IEEE 802.11 are one order of magnitude different. The IEEE 802.11 data shows lowest local transitivity and highest path length. Noting that $59 \%$ of the IEEE 802.11 contributors are disconnected nodes the longer average path length indicates sparse networking and longer path for information sharing even in the connected part of the SSO network.

Table 3. Number of different types of contributors in the SSOs. Comparable data in this table covers the years 2003-2008.

\begin{tabular}{|l|c|c|c|c|c|}
\hline & OMA & 3GPP & IETF & W3C & IEEE 802.11 \\
\hline ALL Contributors $\left(\mathbf{N}_{\text {sso }}\right)$ & 248 & 415 & 1768 & 267 & 294 \\
\hline * Competing only & 40 & 83 & 187 & 12 & 172 \\
\hline * Competing and Collaborating & 173 & 291 & 1038 & 120 & 117 \\
\hline * Collaborating only & 35 & 41 & 543 & 135 & 5 \\
\hline
\end{tabular}


Figure 2. Collaboration profiles of the SSOs. Ratio of Collaboration only (Collaboration $=1$ ), Competitive only (Collaboration=0) and Coopetitive $(0<$ Collaboration<1) companies is shown separately for each SSO. Data consolidated over the research period 2003-2008.

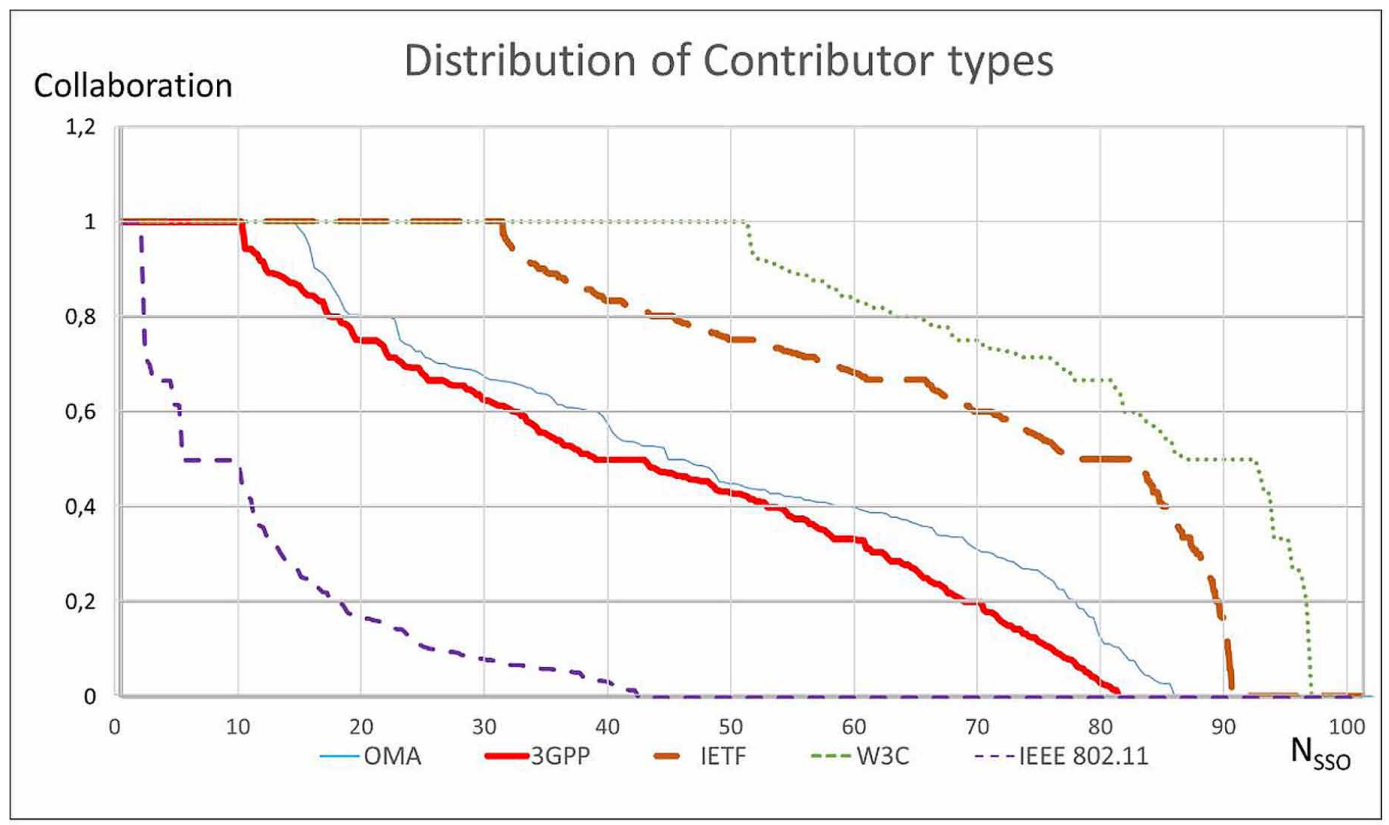

Figure 3. Transitivity and path length. Only connected contributors included (2003-2008).

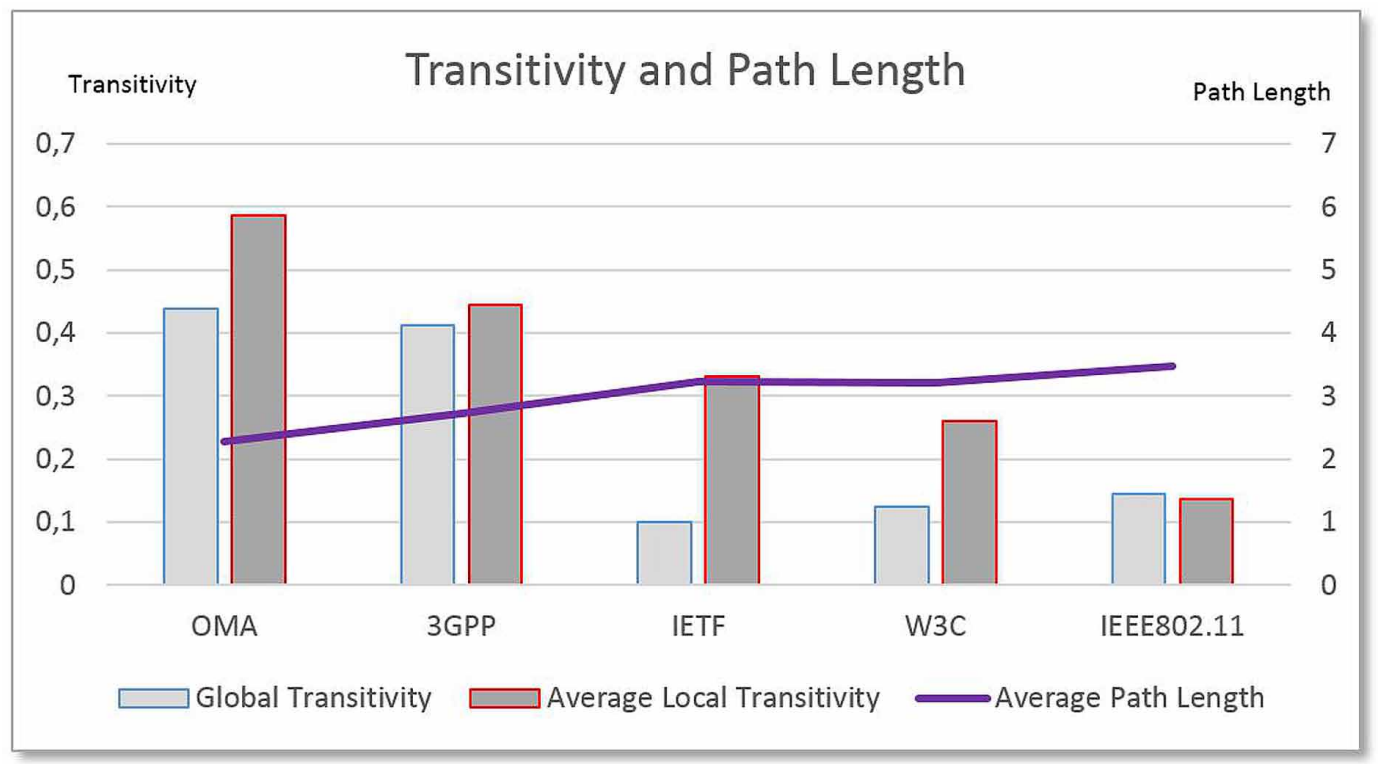




\section{Structures of the Collaboration Networks}

Analysis of network structure utilises two slightly different approaches: degree centrality and eigenvector centrality. Degree centrality is a measure of local networking showing the number of connected nodes for each network node. Degree centrality is an indicator of the network structure for information sharing. In many collaboration networks, the degree centrality distribution often follows the power law. For structural analysis, the number of similar events do not count i.e. all the non-zero link weights equal 1 . The contributor ranking is according to their centrality scores.

Distributions of unweighted degree centrality rankings follow two different trends shown in Figure 4 and Table 4. For 3GPP and OMA, the distribution trend-line is exponential while for IETF, W3C and IEEE 802.11 the trend line follows power law. Tested trend line options were linear, logarithmic and exponential as well as power law. The highest $\mathrm{R}^{2}$ value indicates best fit. Direct ranking of weighted degrees is used instead of the traditional degree distribution to make the curves comparable to a relevant earlier research (Crespo et al., 2014). Local networking in 3GPP and OMA for information sharing shows rather flat, equally shared distributions especially for the top 40 to 60 contributors while in the other SSOs there are only few strongly networking contributors. Edges of the networks are weakly connected in all SSOs.

Figure 4. Unweighted degree centrality scores of all connected contributors in ranking order in the SSOs. Research period 2003-2008.

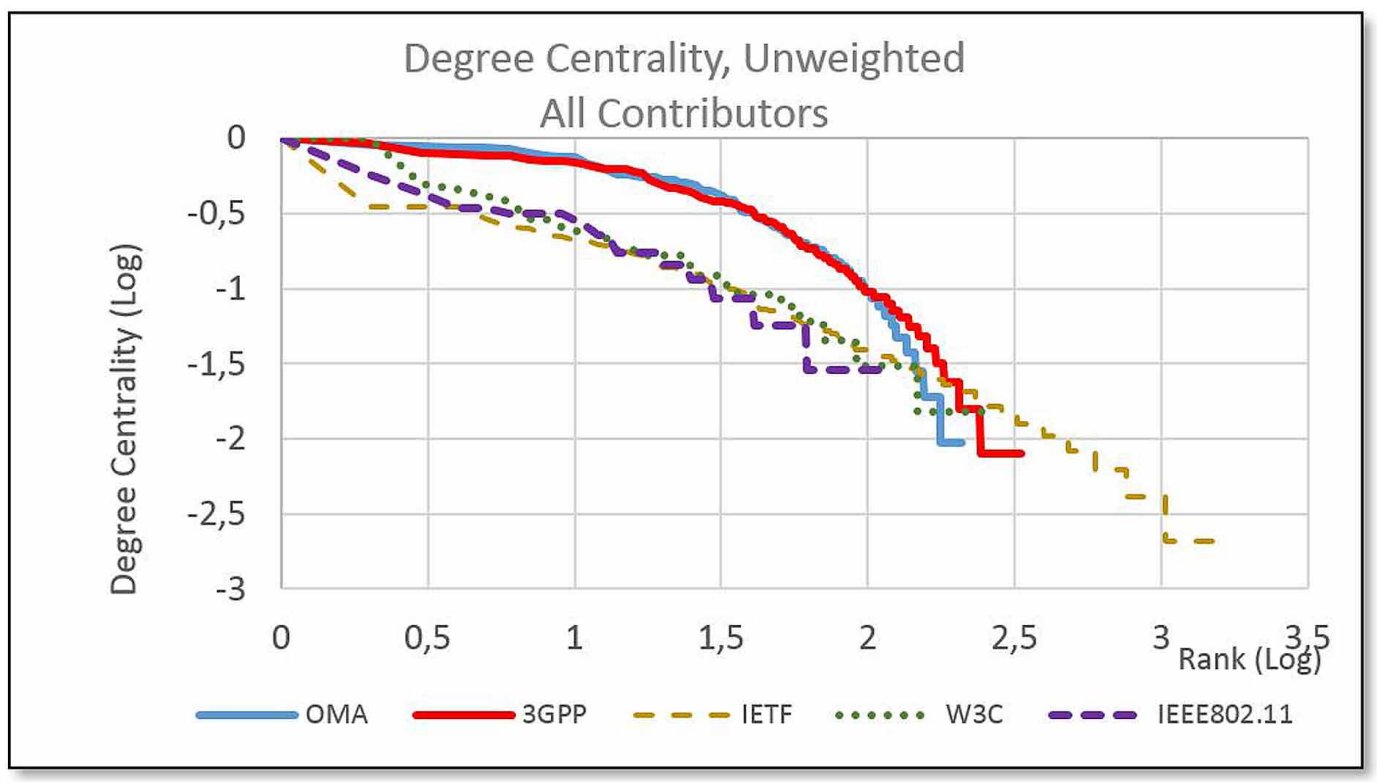

Table 4. Trend line factors, all connected, unweighted degree centrality

\begin{tabular}{|l|c|c|c|c|c|}
\hline & OMA & 3GPP & IETF & W3C & IEEE 802.11 \\
\hline Power law exponent & & & $\mathbf{- 1 , 0 6 4}$ & $\mathbf{- 0 , 9 4 4}$ & $\mathbf{- 0 , 8 9 1}$ \\
\hline Exponential exponent & $\mathbf{- 0 , 0 2 3}$ & $\mathbf{- 0 , 0 1 5}$ & & & \\
\hline $\mathrm{R}^{2}$ & 0,99 & 0,97 & 0,95 & 0,95 & 0,94 \\
\hline Number of connected nodes $(\log ($ degree $>0))$ & 208 & 332 & 1581 & 251 & 122 \\
\hline
\end{tabular}


Eigenvector centrality extends the analysis to the whole network and as a global centrality metric, it captures the global influential power of collaborating standardization contributors. Eigenvector centrality takes all the unweighted connections into account similarly ${ }^{24}$ as the page rank algorithm calculates the centrality of documents in the WWW.

The eigenvector centrality analysis indicates even more clearly that the SSO collaboration styles fall into two different groups (see Figure 5 and Table 5). Furthermore, it shows that the global collaboration is broader than local (slope is slower) but also that the cut off is steeper. This is an indication that practices of the information sharing and influencing are different and that they are different between the two groups. Influencing for decision-making in mobile communications collaboration is more equally distributed than in the internet-oriented forums. For all SSOs, the long tails of the contributors, i.e. edges of the networks are structurally non-influential in this analysis.

The complete set of eigenvalues of the adjacent matrix forms the spectrum of network. Eigenvector centrality takes into account only the information related to the largest eigenvalue (see Table 6). Therefore, the quality of eigenvector centrality as a characterizing metric depends on the relative size of the first eigenvalue to the other eigenvalues. High relative size indicates that the eigenvector centrality scoring is able to capture a significant amount of collaboration dynamics into the first vector. For OMA and 3GPP distance between the largest and second largest is about 1 to 1/3 while in case of IETF, W3C and IEEE 802.11 the distance is about half of that.

\section{Collaboration Activity in the Networks}

Collaboration activity is visible in weighted networks. Weighted degree centrality provides a collaboration activity indication on the top of the network structure (see Figure 6 and Table 7). Link weights reorganize the distribution emphasizing the number of joint contributions, additionally to the number joint collaborators. Weighted degree centrality records all collaborative information sharing but still excludes stand-alone contributions. Exponent of the trend line gives an indication of the hierarchy of activities.

The trend lines for the weighted degree centrality rankings show mainly exponential distributions while a power law distribution was expected. However, a simple regression when applied to all contributors does not necessarily capture all the shades of grey. For the top 40 contributors power law behaviour becomes gradually dominant in IETF, W3C and in IEEE 802.11 while 3GPP and OMA continues to show exponential distribution. Impact of the long tail of contributors is therefore different for information sharing in each different SSO.

Role of any specific contributing organizations is not in the scope of this study. However, in order to connect this research to practice the list of top 5 most active contributors based on the total number of all contributions (stand-alone and collaborative contributions) as well as the contributors with highest score of in weighted degree centrality (collaborative contributions only) is provided in the order of rank separately for each SSO (see Table 8).

\section{Collaboration Style in the Networks}

Degree correlation, also referred as assortativity provides a behavioural information on the collaboration. Assortativity is a kind of collaboration style indicator and it is used e.g. to assess maturity of industrial organizations. For instance, social networks of scientists and academics often show positive correlation while industrial networks are disassortative, showing negative correlation. Negative degree correlation indicates that the collaboration mainly takes place between companies having opposite activity scores. Active, typically large companies are connected with strong ties to smaller or at least less active companies, typically their subcontractors, while having weaker connections to their equal size peers. Link weights apply when calculating assortativity in order to capture the collaboration behaviour beyond the structure. 


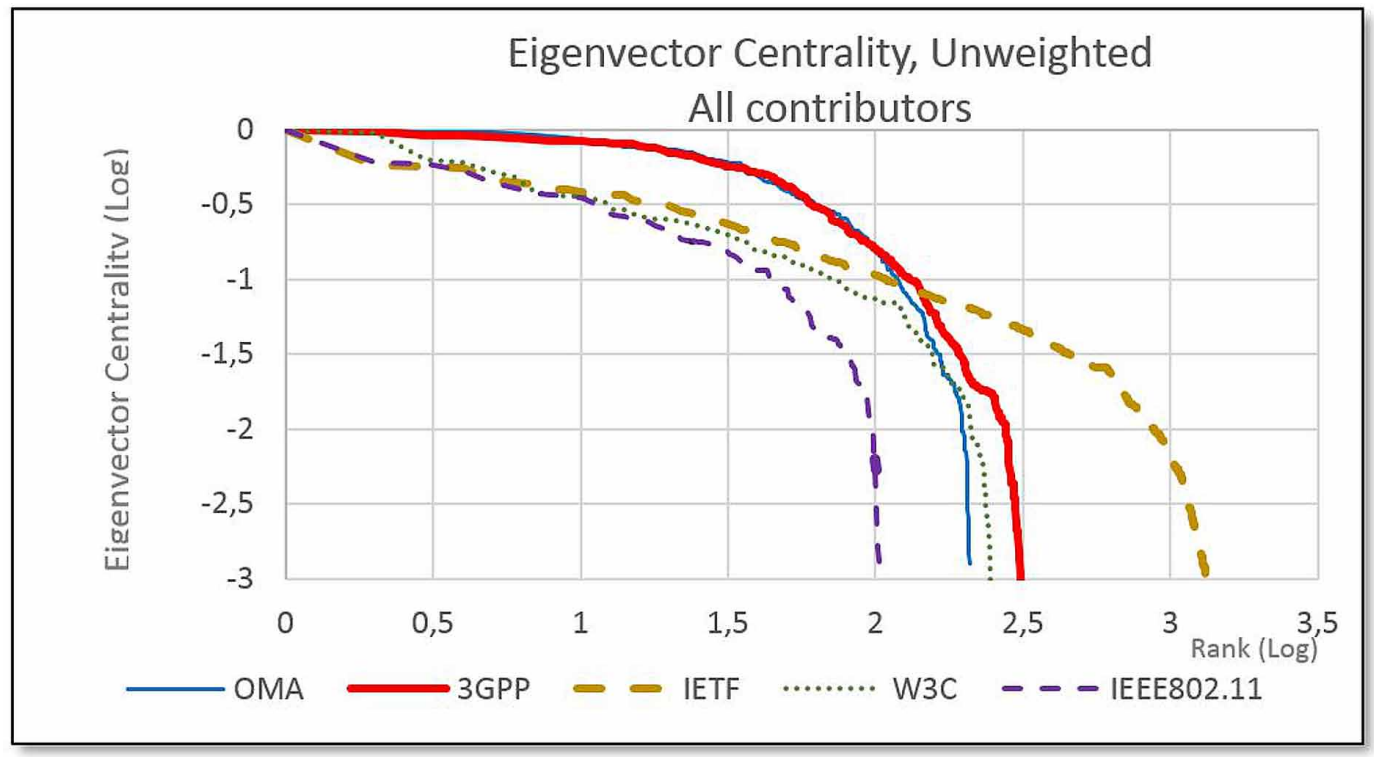

Table 5. Trend line factors, top 40 contributors, unweighted eigenvector centrality

\begin{tabular}{|l|c|c|c|c|c|}
\hline & OMA & 3GPP & IETF & W3C & IEEE 802.11 \\
\hline Power law exponent & & & $\mathbf{- 0 , 3 9 2}$ & $\mathbf{- 0 , 5 1 7}$ & $\mathbf{- 0 , 5 9 1}$ \\
\hline Exponential exponent & $\mathbf{- 0 , 0 1 7 4}$ & $\mathbf{- 0 , 0 1 6 8}$ & & & \\
\hline $\mathrm{R}^{2}$ & 0,98 & 0,99 & 0,97 & 0,98 & 0,97 \\
\hline Number of connected nodes $(\log ($ degree $>0))$ & 40 & 40 & 40 & 40 & 40 \\
\hline
\end{tabular}

Assortativity in IETF shows long time evolution from noisy positive scores gradually to stable negative scores (see Figure 7). The crossover point in this development matches with the timing of the major organizational change in IETF discussed in chapter 3. Even if this event is outside of the focus period of this research, it is a useful observation connecting the assortativity to historical events of IETF. 3GPP shows similar but much more rapid stabilization of the assortative scoring. OMA has a short history with no real changes and W3C shows development from rather strong negative towards less negative scores. IEEE 802.11 shows clearly different behaviour. Instead of rather steady development towards more mature industry collaboration, IEEE 802.11 shows significant cycling with the period of 3-5 years. The collaboration in the IEEE 802.11 cycles between strongly assortative and disassortative scores.

Earlier research has shown that the level of network assortativity gives a good indication of the way knowledge flows between the central and more peripheral nodes (Crespo et al., 2014). Landscape of hierarchy versus assortativity gives a visual indication of resilience in these networks. Figure 8 shows the difference between random, assortative (core-periphery) and resilient network types. All forums show characteristics of resilience by having negative slope in the degree centrality ranking and negative assortativity (note the absolute value for degree centrality lal in the drawing, positions 
Table 6. Relative top 3 eigenvalues of the connected contributor networks of the SSOs

\begin{tabular}{|l|l|l|l|l|l|}
\hline \multicolumn{1}{|c|}{ Relative Eigenvalues } & \multicolumn{1}{c|}{ OMA } & \multicolumn{1}{|c|}{ 3GPP } & \multicolumn{1}{c|}{ IETF } & \multicolumn{1}{c|}{ W3C } & \multicolumn{1}{c|}{ IEEE 802.11 } \\
\hline \# 1 & 1 & 1 & 1 & 1 & 1 \\
\hline \# 2 & 0,28 & 0,31 & 0,53 & 0,60 & 0,62 \\
\hline \# 3 & 0,24 & 0,28 & 0,45 & 0,51 & 0,53 \\
\hline
\end{tabular}

Figure 6. Weighted degree centrality of the connected collaborating companies in ranking order in the SSOs during the research period $2003-2008$

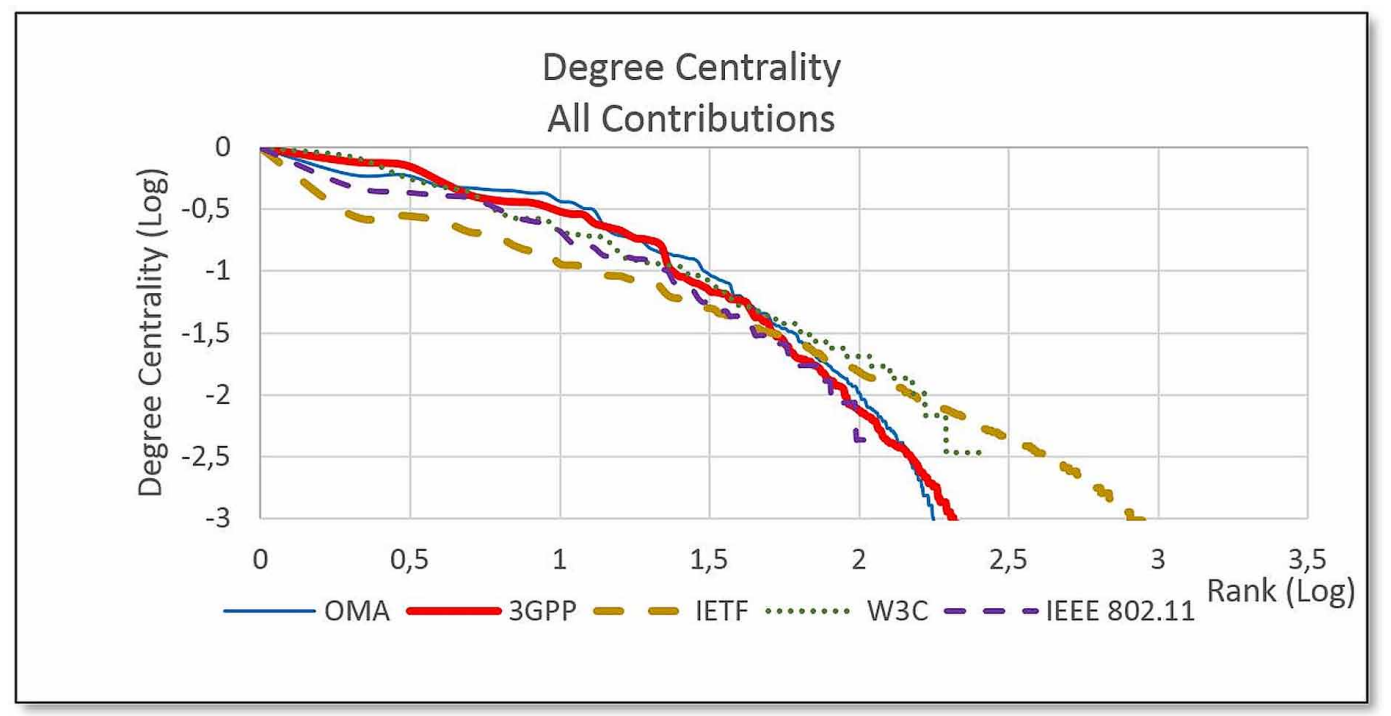

Table 7. Trend line factors, all contributors, degree centrality

\begin{tabular}{|l|c|c|c|c|c|}
\hline & OMA & 3GPP & IETF & W3C & IEEE 802.11 \\
\hline Exponential exponent & $\mathbf{- 0 , 0 3 3}$ & $\mathbf{- 0 , 0 2 3}$ & $\mathbf{- 0 , 0 0 3}$ & $\mathbf{- 0 , 0 1 7}$ & $\mathbf{- 0 , 0 3 8}$ \\
\hline $\mathrm{R}^{2}$ & 0,98 & 0,97 & 0,94 & 0,92 & 0,95 \\
\hline Number of connected nodes $(\log ($ degree $>0))$ & 208 & 332 & 1581 & 255 & 122 \\
\hline
\end{tabular}

of the SSOs are illustrative only). The assortativity cycling in IEEE 802.11 makes the SSO to cycle also in this landscape model between the two positions of resilient and core-periphery behaviours.

\section{SEMI-Quantitative Visualization of the Collaboration Networks}

As the last analysis method, maximum spanning tree shows visually the network topology and gives further insights to the collaboration in standardization. The spanning trees in this paper use all the same scale where the size of circles indicates the total number of contributions and the width of links indicates the number of joint contributions between the nodes. The maximum spanning tree shows only the critical links of collaboration. Additionally, maximum spanning tree supports the semiquantitative high-level validation of input data when trees are used parallel to the interview findings. Maximum spanning trees drawings support qualitative assessments regarding disconnected nodes, 
Table 8. Top five contributors in the order of rank based on the number of contributions and separately based on their weighted degree centralization scoring in the SSO. Comparable data available for the period 2003-2008.

\begin{tabular}{|l|l|l|l|l|l|l|l|l|l|}
\hline $\begin{array}{c}\text { OMA } \\
\text { Activity }\end{array}$ & \multicolumn{1}{|c|}{$\begin{array}{c}\text { OMA } \\
\text { Degree }\end{array}$} & $\begin{array}{c}\text { 3GPP } \\
\text { Activity }\end{array}$ & $\begin{array}{c}\text { 3GPP } \\
\text { Degree }\end{array}$ & $\begin{array}{c}\text { IETF } \\
\text { Activity }\end{array}$ & $\begin{array}{c}\text { IETF } \\
\text { Degree }\end{array}$ & $\begin{array}{c}\text { W3C } \\
\text { Activity }\end{array}$ & $\begin{array}{c}\text { W3C } \\
\text { Degree }\end{array}$ & $\begin{array}{c}\text { IEEE 802.11 } \\
\text { Activity }\end{array}$ & $\begin{array}{c}\text { IEEE 802.11 } \\
\text { Degree }\end{array}$ \\
\hline Ericsson & Nokia & Ericsson & Nokia & Cisco & Cisco & W3C & W3C & Intel & Intel \\
\hline Nokia & Ericsson & Nokia & Ericsson & Ericsson & Ericsson & IBM & IBM & Cisco & Cisco \\
\hline Huawei & Huawei & Huawei & NSN & Nokia & Nokia & Microsoft & Microsoft & Motorola & Boeing \\
\hline Samsung & Orange & NSN & Nortel & Nortel & Nortel & Sun & Sun & Broadcom & Air Wave \\
\hline Motorola & Qualcomm & Siemens & Huawei & Microsoft & Juniper & HP & HP & Boeing & Marvell \\
\hline
\end{tabular}

Figure 7. Assortativity evolution in the SSOs. Comparable data available for the period 2003-200825.

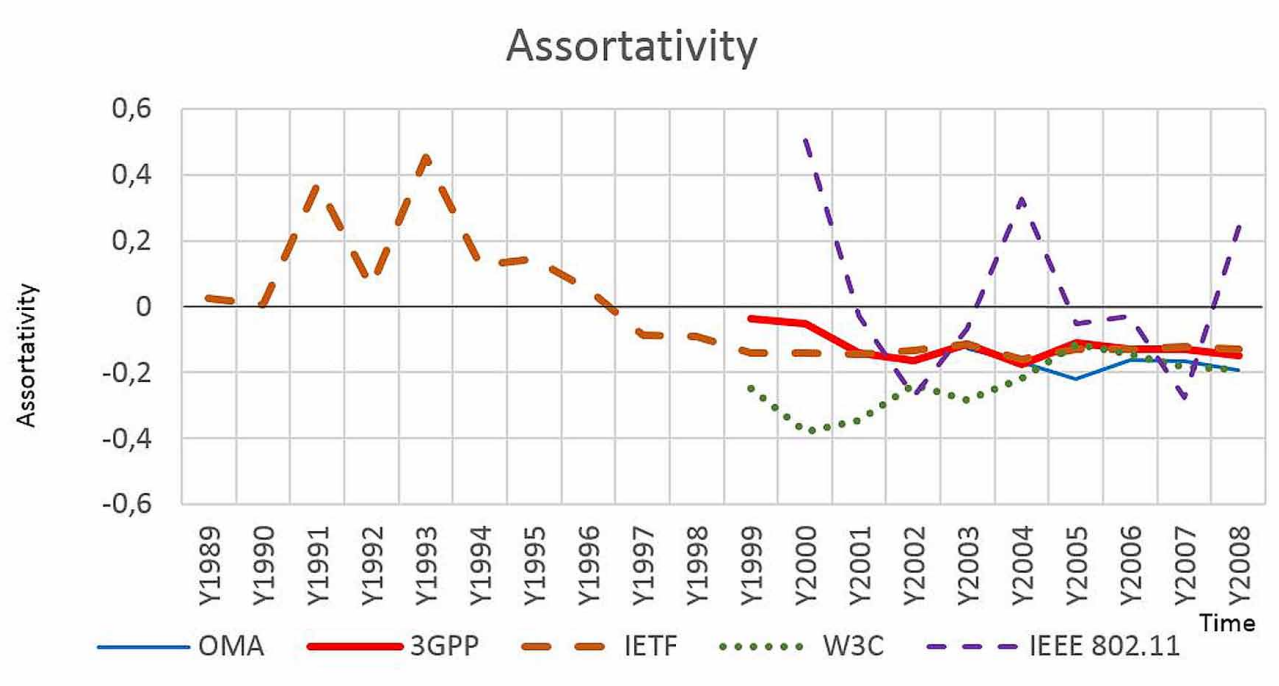

Figure 8. Resilience of the collaboration modes of the SSOs (adapted from (Crespo et al., 2014))

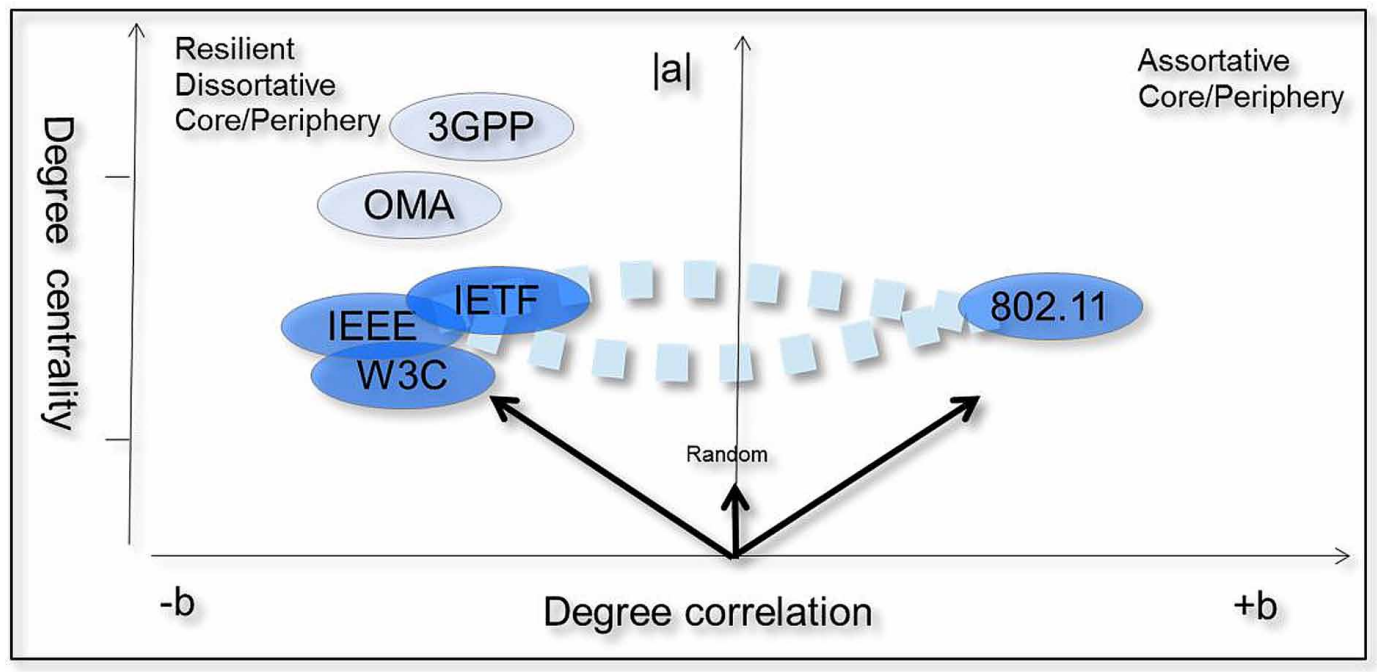


as those are visible in the same picture. It is possible to study e.g. globalization and collaborating strategies of the contributors by observing changes in the trees over time. Time series of spanning trees also show changes in the roles of the companies over time.

In the 3GPP spanning tree in Figure 9, the most active contributors connect to each other with quite strong links and each of the key contributor connects a separate sub-network of contributors to the main network. The 3GPP spanning tree shows rather clear logical grouping of the contributors based on the type of actors and their combined contribution activity of each branch. The top five active contributors are listed in Table 7. Group A: Mainly Network operators, Group B: Mainly Korean companies, Group C: Mainly Japanese companies, Group D: Mainly Chinese companies, Group E: Mainly technology and equipment vendors. The stand-alone contributors form a disconnected cloud outside of the connected network.

As another example using the same methodology as in Figure 9, Figure 10 shows the IEEE 802.11 maximum spanning tree. It is different in many ways. The total number of contributions is much smaller than in 3GPP as is visible in the size of circles. The high number of stand-alone contributors forms a large cloud outside of the main connected network. Branches of the tree include companies without any specific grouping logic. These observations are aligned with other studies related to standardization in telecom and internet oriented standardization communities (Jakobs et al., 2001), (Isaak, 2006).

\section{DISCUSSION}

The SNA provides insightful perspective on the internal collaboration in each of the five SSOs. The semi-structures interviews complement the quantitative results by providing views, which in most cases align fully with the SNA findings with some exceptions as discussed below.

\section{GPP}

The maximum spanning tree of the 3GPP collaboration network gives an indication of the importance of historical evolution when companies with the same geographical and regulative origin, China, Korea and Japan show up each in their own branch of the tree. Like-minded companies, like majority of the

Figure 9. Maximum spanning tree of the 3GPP collaborative network consolidated over the years 2003-2008

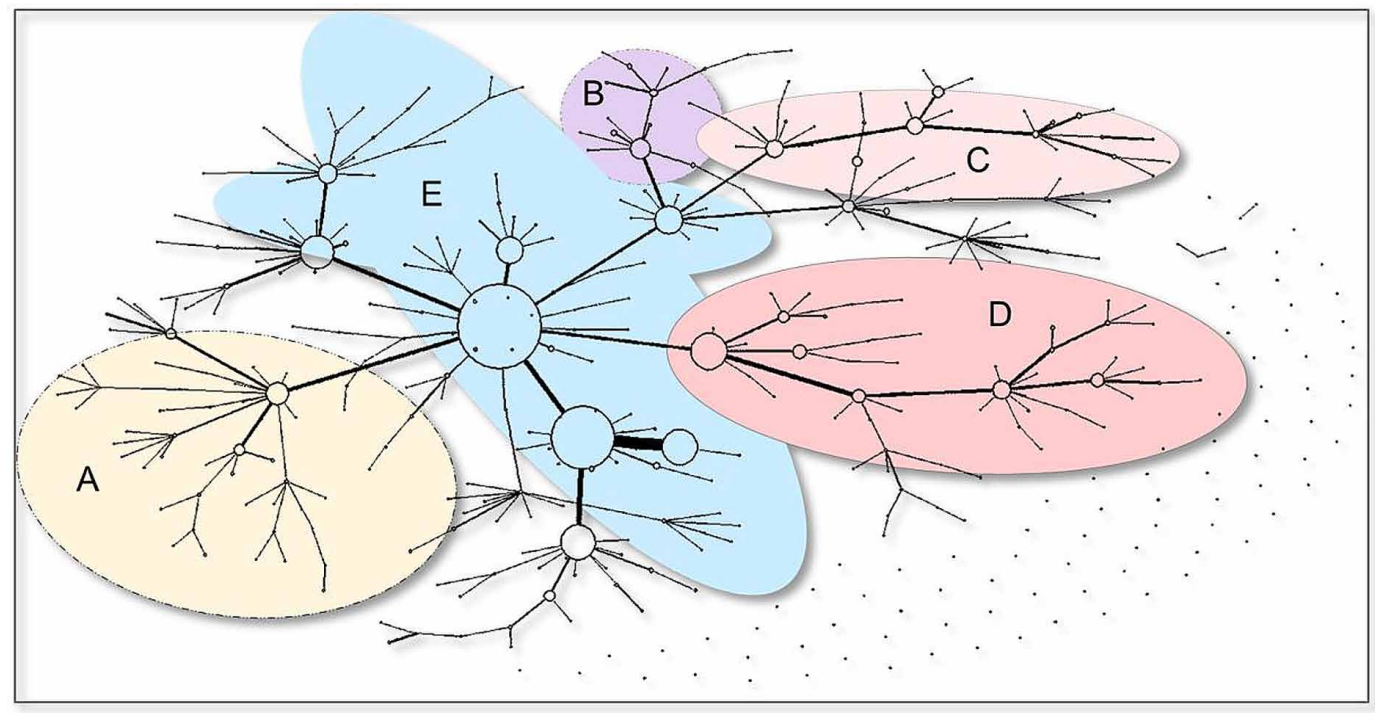




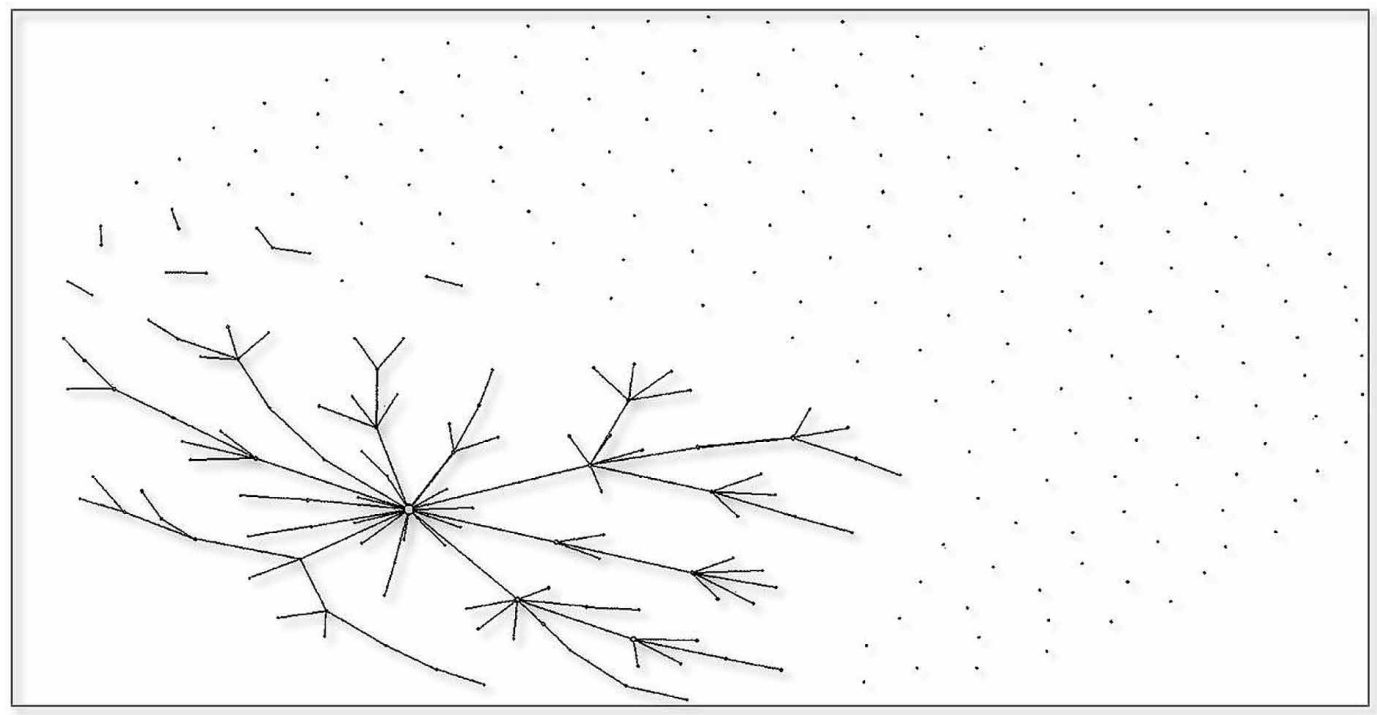

network operators form up one main branch of the maximum spanning tree while the main body consist of technology and equipment vendors. The interviews note the presence and specific role of licensed network operators in 3GPP. The operators can shape the collaboration in 3GPP individually and as a group through their global and regional collaboration organizations such as the GSM Association (GSMA) and the GSM operators in North America (GSM NA). "The role of the operators is much more direct and strong than for instance the role of the PCG (Project Coordination Group)....The GSMA however does not always integrate the opinions of all the operators" as one of the 3GPP expert explained. Mobile network operators have local natural monopoly due to the licensed radio spectrum their networks utilize. The maximum spanning tree analysis shows the network operators to form their own branch rather than positioning them to the middle. This reflects their role as a significant but not as the most active contributor group.

A specific characteristic discovered in the structure of 3GPP network is that both degree centrality and especially eigenvector centrality show a rather flat distribution of the global information sharing and influence indicating broad and rather equal participation by a large number of contributors to the discussions and decision-making in 3GPP. Weighted degree centrality distribution follows the shape of the unweighted distribution but the slope is steeper indicating that hierarchy in the collaborative actions is higher than in the structure. This means that a smaller group of companies provides large part of the joint documents.

These observations are intuitively well in-line with the strong systemic interoperability requirements of the inherited formal standardization environment of 3GPP including regulated, mainly national roles of the network operators. Interviewees noted the heritage of historical roots of the SSOs. One 3GPP expert explained the path dependency from ETSI to 3GPP. "It took several years in ETSI to leave the traditional CEPT mode of operation behind. The situation only changed when the market changed and all participants had to start working according to their new market position. Similar difficulties were experienced when $3 \mathrm{GPP}$ was set up. It took several years for participants from the different markets to adopt the new international way of working." When eigenvector centrality is studied over years the development towards broader collaboration can be seen as the curve becomes more and more flat over the years (from 2003 to 2008) (not shown in this paper). 
Speed of technology development in the mobile communications is fast. Lifetime of one generation of technologies is shorter that lifetime of the spectrum license. Large variations of product and service deployments within the operator community together with strong expectation on global roaming have consequently created strong backwards compatibility requirement for the 3GPP technologies and networks. Such a complicated network architecture and large number of interoperable interfaces obviously benefit from the stability of the collaboration mode. After initial formation period, negative assortativity with rather high clustering and hierarchy indicates stable and resilient operative mode in 3 GPP over the timeframe of this study. The initial ambiguous phase of collaboration is weakly visible as the assortativity of the 3GPP contributors stays close to zero until 2001, more than 3 years after the ETSI decision in 1998 to use WCDMA technology for the 3rd generation network was made. One of the 3GPP experts commented: "Japanese delegations did not understand in the beginning how the system worked and there were top level people trying to influence using interpretation (ITU Style) but that was soon taken over by English speaking technical experts and the 3GPP working style was adopted".

\section{OMA}

The similarities between OMA and 3GPP are strong in all aspects studied in this research. The fact that the service enablers OMA develops have no license dependent or regulation-oriented requirement show no reflections in the way the OMA collaboration is structured. As it relates to OMA, the maximum spanning tree constellation provides useful semi-quantitative insight to the structure of collaboration network. Using the same approach as in Figure 9 for 3GPP, the following groups become visible. Group A: Mainly network operators, Group B: Mainly equipment vendors, Group C: Mainly IT companies and Group D: Mainly Chinese companies.

Yearly trees show strong network operator influence in the beginning followed by a growing role of equipment vendors and finally by the year 2008 the equipment and technology vendors including also new entrants from Far East, especially from China, dominate the collaboration in OMA. The IT companies tried to seek collaboration with a number of other contributors instead of systematically building their own cluster. However, the consolidated data shows the IT companies forming a small cluster of their own in the Figure 11.

\section{Figure 11. Maximum spanning tree of the OMA collaborative network consolidated over the years 2003-2008}

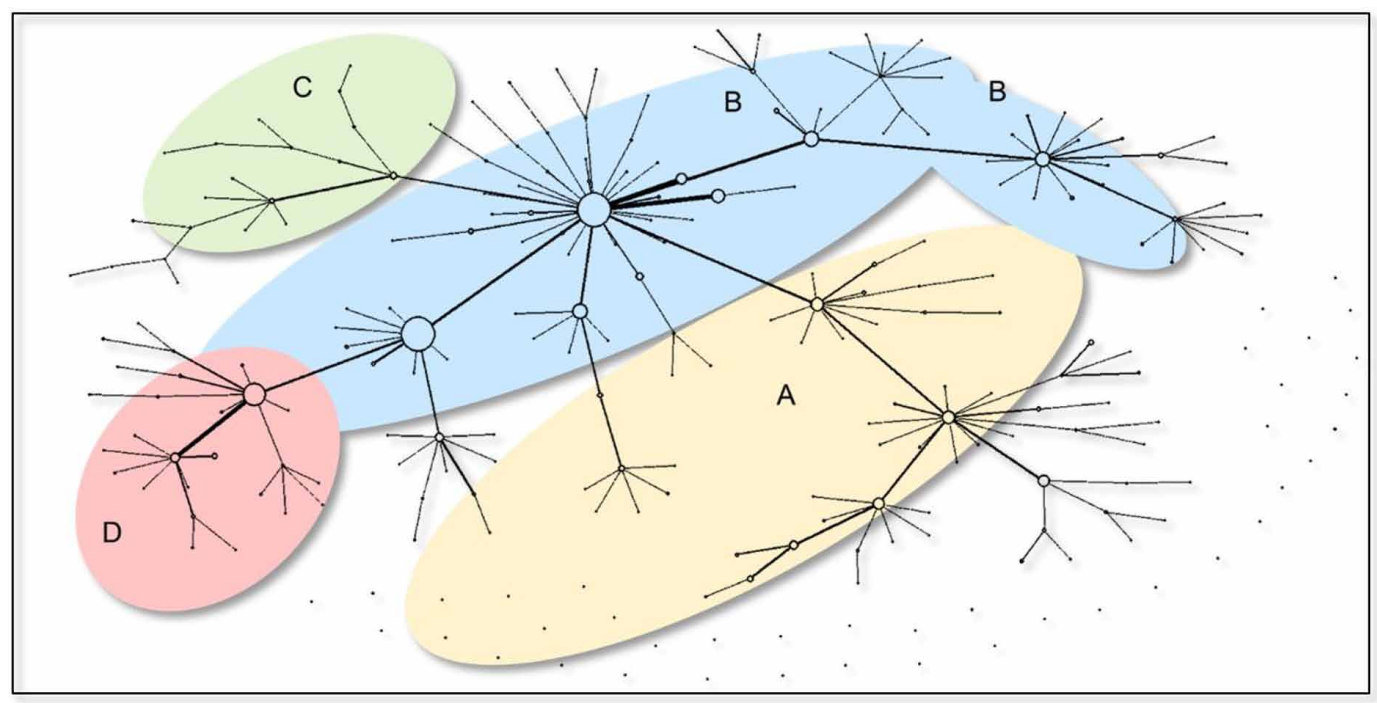


The role of IT companies was seen stronger in some interviews than what can be observed in the maximum spanning tree. This may reflect a good intention to bring the IT companies into the mobile communications standardization but at the same time, it shows the difficulties on practical level. "In OMA it was decided in very early on to involve all the key parties, telecom operators, equipment manufactures and IT companies. The Board consisted of these 3 baskets, and the CDMA companies. The board and technical plenary were separated to let the technology decisions be based on technical merits only", explained one ex-member of the OMA board. The specific role of Chinese companies was not foreseen at all when OMA was set up.

The collaboration style in OMA shows stable industrial behaviour from the beginning. This is in-line with the conscious decision-making in the formation process of OMA.

\section{IETF}

The Internet oriented SSOs are working with technologies for businesses with less regulatory constraints. IETF collaboration structure shows power law distribution both in degree and eigenvector centrality. IETF is less hierarchic than 3GPP and OMA but on the other hand, the role of the leading company is significant both locally as a very active contributor and globally as a strong influencer throughout the network. Separately, a 3GPP standardization expert working also in IETF noted the importance of the leading individuals in the consensus building: "The significant role of strong individuals can make big things in IETF. In 3GPP, technical arguments are more powerful while in IETF the gurus are very powerful. “

The very high number of collaborating companies is visible in the network, i.e. the long tail contributors are steadily connected to the main network, and IETF has a low percentage of standalone contributors. The IETF transition from a community to a solid industrial organization took place around 1997 and therefore during the research period the IETF collaboration style is stable.

\section{W3C}

When W3C was established, the membership rules encouraged a large number of participants to join. This caused later a rather high churn in the delegate community. The long tail of degree centrality (weighted and unweighted) reflecting the open access rules is clearly visible. The tails of W3C as well as IETF distributions are in relative terms longer when compared to the tails of the OMA and 3GPP distributions. The collaboration profiles and separately the long tail of contributor distributions show similarities between IETF and W3C.

The W3C collaboration shows strong disassortative behaviour from 1999 onwards. The available data covers only the period from 5 years after the formal set up of the organization. The strong negative assortativity is visible in the maximum spanning trees on yearly level also where the trees break down to several separate smaller networks, kind of spanning bushes. This may be an indication of a strong competition between the different browser technologies during those years.

\section{IEEE 802.11}

The collaboration in IEEE 802.11 shows structural similarities to other Internet oriented SSOs, IETF and W3C. However, IEEE 802.11 is different from all the other SSOs in two aspects. The collaboration profile shows the high proportion of stand-alone contributors and the assortativity scores show the strong variation over time. Both are unique features of the IEEE 802.11 collaboration requiring further discussion.

The high number of contributors providing only stand-alone contributions leaves the collaborating contributors in minority. The collaboration approach of this minority however, follows the patterns of other Internet oriented SSOs regarding network clustering as well as in degree and eigenvector centrality. By observing the circle sizes in the IEEE 802.11 collaboration spanning tree it is rather clear that the connected contributors are also the most active contributors while the large cloud of disconnected nodes consists of less active contributors. The SNA results reflect the behaviour of the 
most active contributors in IEEE 802.11, as is the case for other SSOs also. As brought up by one IEEE 802.11 expert during the interview, engineers personally interested in a technology and seeking to promote it for broader use are driving the collaboration in IEEE 802.11. The weak and unstructured maximum spanning tree of IEEE 802.11 therefore is likely to indicate focused technology orientation of the contributors. The traditional "individual" membership makes the difference to the way the SSO is operating as noted in the earlier research also (Lemstra, Hayes, \& Groenewegen, 2011).

The oscillating assortativity of the IEEE 802.11 collaboration style is quite an important discovery. All other SSOs show only steady negative assortativity scores during the research period. The strong temporary positive scores in the IEEE 802.11 collaboration need special attention. The cycling seems to synchronize with the major undertakings in the IEEE 802.11 community. The slopes towards negative peaks in 2002 and 2007 and for the positive peaks in 2000, 2004 (and 2008) align with the technology selection and finalization phases of the IEEE 802.11 releases of $11 \mathrm{~g}, 11 \mathrm{n}$, respectively ${ }^{26}$ (see Figure 12).

When observing the development of the IEEE 802.11 maximum spanning tree parallel with the cycling assortativity it becomes apparent that for each cycle the leading contributors seem to focus first on the concept and system level parameters making the assortativity positive. Later in the process when details are defined, resilient collaboration is needed and assortativity becomes negative. The interviews emphasize the market orientation as a critical success factor for any standardization, including IEEE 802.11. Low presence of end user level customers, like network operators or Wi-Fi access network owners facilitates the specific collaboration characteristic of the IEEE 802.11 group. Furthermore, this observation correlates with a comment by one interviewee that the deployment of the IEEE 802.11 standardized technologies and products require only local decisions. In commercial applications, e.g. in corporations, Wi-Fi networks can be upgraded at the same pace with laptop computer population. Therefore, each new product renewal cycle is a strong driver in standardization. Backward interoperability and roaming capabilities in Wi-Fi networks have not been very critical

Figure 12. Aligning IEEE 802.11 standards releases and the assortativity of the collaboration

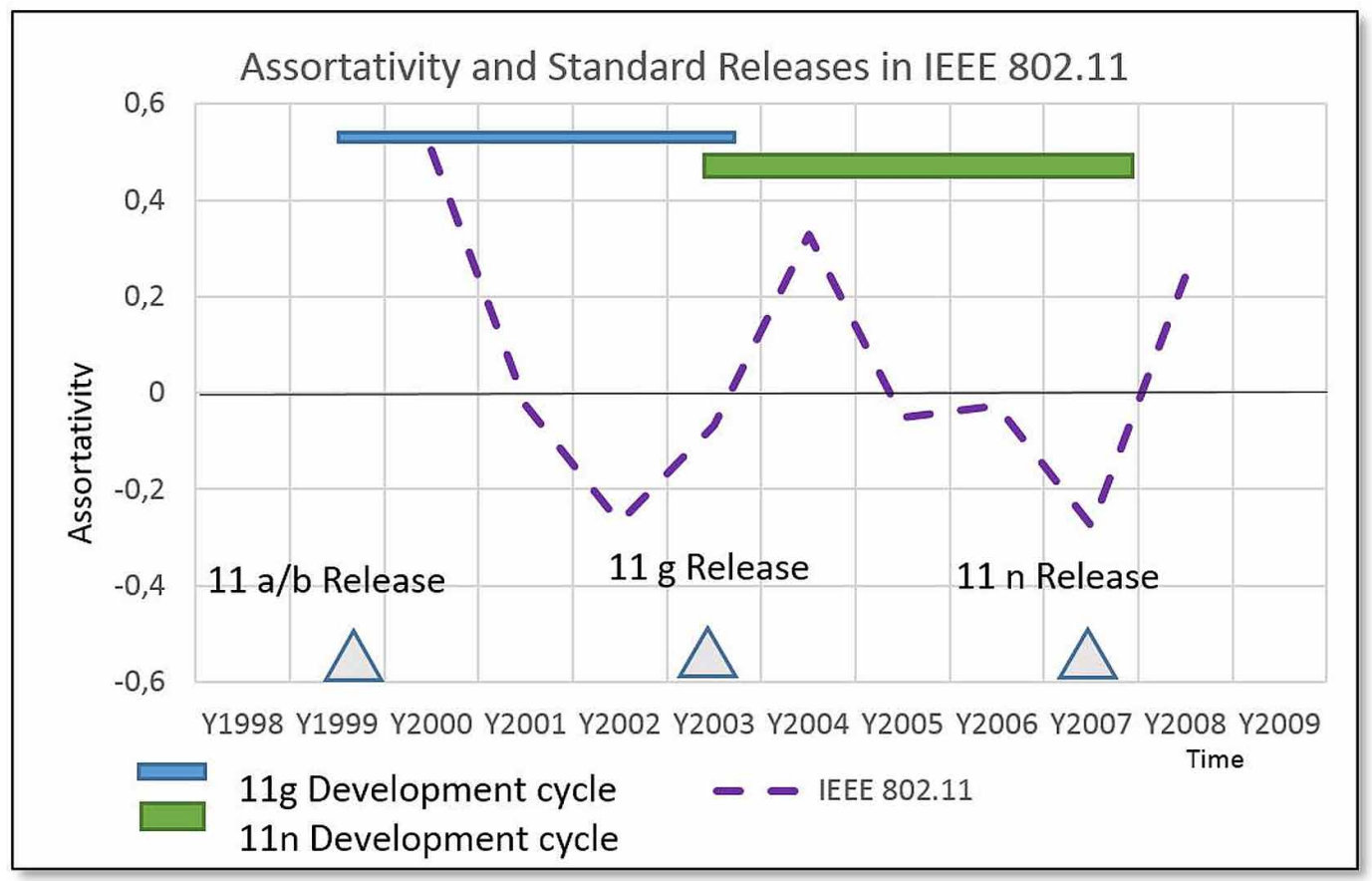


features, especially during the years 2003-2008. The Wi-Fi comes as a by-product of the computer industry (Lehr \& McKnight, 2003). The less coordinated nature of the IEEE 802.11 process has, however, caused several hiccups and glitches during the lifetime of the group (Kaa van de \& Bruijn de, 2015). Even if there are multiple parallel activities in IEEE 802.11, the main releases of the new Wi-Fi radio technologies set the pace to the overall collaboration.

In the other SSOs similar cycling of collaboration mode is not observed as the groups steadily maintain the negative assortativity. The factors however, behind the observation may be different for each SSO. The interviews related to $\mathrm{W} 3 \mathrm{C}$ emphasize the deliberate decision to avoid any specific standard release cycles due to the possibility to download the software implementations of web browsers and servers using the W3C standards as the new versions become available. The same is valid for IETF standards into large extend. Furthermore, the large number of working groups each with their own schedules makes the assortativity to show the average scores only. For 3GPP, where the ridged releases are used the assortativity still does not cycle. 3GPP operative mode covers the development of multiple system level releases at the same time with strong backwards and forwards interoperability requirements. There is no possibility to develop any system standard fully release independently from other releases ${ }^{27}$. The standards development work is in a continuous flow. Originally, OMA was also aiming at full system releases but soon it became evident that it was impossible and each OMA work item got a permission to proceed according to their own schedule. All these factors require mind-set of continuous development and therefore assortativity remains stable over the years. An analysis addressing subgroup level collaboration is for further study.

As discussed in chapter 3 the WFA promotes the deployment of the Wi-Fi products through the certification process and therefore part of the decision-making related to the Wi-Fi standardization is outside of the IEEE 802.11 group. In other words, the Wi-Fi community sets standards using consecutive technology definition gates as indicated by the assortativity analysis and by observations on the process from standards through certification and market making to commercial products ${ }^{28}$. The other SSOs seem to develop standards in a more continuous process and they rely mainly on their internal competences to define the further steps in the process e.g. the certifications related to $3 \mathrm{GPP}$ and $\mathrm{OMA}^{29}$.

\section{Similar and Different}

The visible collaboration is similar in all standardization organizations studied in this research. All SSOs follow the process where initial ideas, requirements and technology capabilities are converted into solid specifications and standards to make the compliant products to interoperate. The contribution process is similar based on written documents provided by the contributors. Some contributors are more active in collaboration and contributions as becomes visible through quantitative analyses. All the SSOs studied are "Small Worlds" of their own.

The SSOs working in an environment where licensed network operators have strong role have harmonized their collaborations structures and styles almost completely. The needs of the licensed network operators drive the standardization in 3GPP and OMA (Brzezinski, 2007). Similar alignment towards another set of characteristics is visible in the environment where such licenses are not necessary. These observations are aligned with earlier literature indicating high likelihood of synchronization of two complex systems with similar dynamics (Strogatz, 2001).

Another high-level differentiator between the SSOs is in their focused component or broad systems thinking mind-set. 3GPP covers all the layers of the mobile communications requiring system level approach. Software based implementation of the standards enables, and also requires IETF, W3C and also OMA to pay attention to interoperability through downloading. IEEE 802.11 stays alone as $\mathrm{s}$ focused physical layer oriented group. The different natures of hardware and software implementations as well as component and system scope motivate the contributing companies with commercial interests in products and services to optimize their strategies to deal with market uncertainties differently in different environments. (Gaynor \& Bradner, 2001). 
The third area of different behaviours relates to the historical roots and traditions to compete and collaborate within these communities. The level of collaboration falls into three groups where IETF and W3C form one group OMA and 3GPP form another group whereas the collaboration in IEEE 802.11 has its own characteristic. These traditions increase trust and productivity but when some contributors are highly collaborative such a behaviour may also raise questions on free riding. High collaborative attitude however, brings value by increasing stability and consensus. Similarly, some of the stand-alone contributors, after all, may be collaborative even when their contributions in short term may create confrontation.

The key findings of this study are in Table 9.

\section{CONCLUSION}

This research identified three different types of standardization models for mobile communications and internet technologies. The collaboration models in 3GPP and OMA are in full alignment, in IETF and in $\mathrm{W} 3 \mathrm{C}$, the collaboration networks have strong mutual similarities, and the collaboration in IEEE 802.11 has its own character. The results also give strong implications to see the causality between the predefined regulatory framework for the standardization and the implemented approach in each SSO.

The real difference between the SSOs is hiding deep in the collaborative structures and processes, which become visible using the tools like SNA. The SNA and the intuitive observations of the interviews align very well. The specific need of the global structural collaboration, indicated especially by the eigenvector centrality distributions in case of OMA and 3GPP is not easy to measure without SNA tools. Similarly, the fluctuations of collaborative style over time as observed especially in case of IETF and IEEE 802.11 become visible only when using advanced metrics like assortativity. The number of the documents used in the quantitative analyses is rather high and meaningful differences become visible only by reducing redundant information wisely. In standardization, every company is connected to every other company in large meetings and through even larger email reflectors but the challenge is to find out which of the connections are important. The comparable analysis of the five SSOs aims to find relative differences between the forums rather than absolute values or truths.

When building on the top of the quantitative SNA results the qualitative observations become meaningful. Until today, understanding the way of working in standardization may have been difficult in general but when all the documents in the ICT standardization are downloadable it is possible to run SNA almost in real time and observe very early on if some important changes are about to take place. Similarly, for a company considering joining a collaborative effort understanding the invisible structures of information sharing and influencing might be quite useful. Social network analysis has been applied to many network problems in the past. Standardization collaboration networks have not been extensively studied so far using SNA tools. The results of this study are into large extend aligned with previous SNA based research. Observed deviations will require further work and potentially new algorithms especially for analyses of time variant, dense networks with multiple goals, subgroups and priorities. The SNA is a very promising research approach to study complex collaborative networks.

Standardization professionals might find most of the findings intuitively plausible and encouraging for further collaboration in standardization with holistic and systemic mind-set. Results of this research invite to explore collaboration between SSOs. While visible actions of the contributors in information sharing may be rather similar in all SSOs, the well-known challenges of multi-forum collaboration may become manageable through a deep tacit knowledge on behaviours in each SSO. If the different long term, less visible aspirations as discovered in the assortativity time series or the different needs to collaborate during the standardization process, as visualized in the network structures are not understood, it is likely that the inter-SSO collaboration is vulnerable to several misunderstandings and unrealistic expectations. As a further study the complexities of the inter SSOs activities such as the IMS standardization will be useful. 
Table 9. Key findings of the SNA complemented with qualitative observations

\begin{tabular}{|c|c|c|c|}
\hline & $\begin{array}{c}\text { Mobile } \\
\text { Communications } \\
\text { OMA, 3GPP }\end{array}$ & $\begin{array}{l}\text { Web, Internet } \\
\text { IETF, W3C }\end{array}$ & $\begin{array}{l}\text { Access } \\
\text { IEEE 802.11 }\end{array}$ \\
\hline Collaboration profile & High & Very high & Low \\
\hline Transitivity/Clustering & Very high & High & High \\
\hline $\begin{array}{l}\text { Local activity } \\
\text { Weighted Degree Centrality distribution }\end{array}$ & $\begin{array}{l}\text { Exponential, } \\
\text { Medium tail }\end{array}$ & $\begin{array}{l}\text { Exponential } \\
\text { Long/Medium } \\
\text { tail }\end{array}$ & $\begin{array}{l}\text { Exponential, } \\
\text { Short tail }\end{array}$ \\
\hline $\begin{array}{l}\text { Global structural influencing } \\
\text { Unweighted Degree and Eigenvector Centrality } \\
\text { distribution }\end{array}$ & $\begin{array}{l}\text { Exponential } \\
\text { Medium tail }\end{array}$ & $\begin{array}{l}\text { Power law } \\
\text { Long/Medium } \\
\text { tail }\end{array}$ & $\begin{array}{l}\text { Power law } \\
\text { Short tail }\end{array}$ \\
\hline Assortativity & Negative & Negative & $\begin{array}{l}\text { Cycling positive and } \\
\text { negative }\end{array}$ \\
\hline Regulative framework & Licensed (3GPP) & Unlicensed & License Exempted \\
\hline End customer presence & High & Medium/Low & Low \\
\hline Interoperability Focus & Very high Global & High Global & Primarily Local \\
\hline Scope & System & Network & Access/Component \\
\hline
\end{tabular}

Finally, the implications of this research to the policy makers include encouragement to build the bridge between constraints set by the policies and the expected output of the related standardization initiatives. Without systemic set of rules, including competition and collaboration models, standardization is not able to progress efficiently. Different licensing models while liberating the use of scarce resources require complementing interoperability requirements. This equilibrium includes implications related to governance rules for the applicable standardization community. By understanding the fundamental differences between the forums and groups better, a peaceful and fruitful collaboration become achievable. These learnings will be crucial when ICT based solutions, services and products will spread to and shape gradually all sectors of human life. Furthermore, explicit needs to standardize new interfaces on higher layers of abstraction e.g. data and identity will emerge. In the future standardization peaceful collaboration will be at least as useful and fruitful behaviour than war and direct competition.

\section{ACKNOWLEDGMENT}

Nokia Corporation supported this research by providing access to the raw empirical data. Otherwise, the author is responsible on all the research and results alone. The author wants to thank specifically the anonymous interviewees who provided valuable insights to the collaboration and competition within and between the SSOs. Finally, the author thanks the anonymous reviewers who provided very valuable comments and constructive criticism during the process. 


\section{REFERENCES}

Ali-Vehmas, T., \& Casey, T. R. (2012). Evolution of wireless access provisioning: A systems thinking approach. Competition and Regulation in Network Industries, 13(4), 333-361.

Anderson, C. (2006). The long tail: Why the future of business is selling less of more. New York, NY: Hyperion Books.

Bar, T., \& Leiponen, A. E. (2014). Committee composition and networking in standard setting: The case of wireless telecommunications. Journal of Economics \& Management Strategy, 23(1), 1-23. doi:10.1111/jems.12044

Barabási, A.-L., \& Bonabeau, E. (2003). Scale-Free Networks. Scientific American, (May): 50-59. PMID:12701331

Baron, J., Meniere, Y., \& Pohlmann, T. (2014). Standards, consortia, and innovation. International Journal of Industrial Organization, 36, 22-35. doi:10.1016/j.ijindorg.2014.05.004

Blind, K. (2011). An economic analysis of standards competition: The example of the ISO ODF and OOXML standards. Telecommunications Policy, 35(4), 373-381. doi:10.1016/j.telpol.2011.02.007

Blind, K., \& Gauch, S. (2008). Trends in ICT standards: The relationship between European standardisation bodies and standards consortia. Telecommunications Policy, 32(7), 503-513. doi:10.1016/j.telpol.2008.05.004

Brunsson, N., Rasche, A., \& Seidl, D. (2012). The Dynamics of Standardization: Three Perspectives on Standards in Organization Studies. Organization Studies, 33(5-6), 613-632. doi:10.1177/0170840612450120

Brzezinski, K. M. (2007). Network Operators Requirements and the Structure of Telecommunications Standards. International Journal of IT Standards and Standardization Research, 5(1), 103-117. doi:10.4018/jitsr.2007010102

Cabral, L., \& Salant, D. (2014). Evolving technologies and standards regulation. International Journal of Industrial Organization, 36, 48-56. doi:10.1016/j.ijindorg.2013.07.006

Campbell-Kelly, M., \& Garcia-Swartz, D. D. (2013). The history of the internet: The missing narratives. Journal of Information Technology, 28(1), 18-33. doi:10.1057/jit.2013.4

Choi, H., Kim, S.-H., \& Lee, J. (2010). Role of network structure and network effects in diffusion of innovations. Industrial Marketing Management, 39(1), 170-177. doi:10.1016/j.indmarman.2008.08.006

Crespo, J., Suire, R., \& Vicente, J. (2014). Lock-in or lock-out? How structural properties of knowledge networks affect regional resilience. Journal of Economic Geography, 14(1), 199-219. doi:10.1093/jeg/lbt006

Crespo, J., Suire, R., \& Vicente, J. (2016). Network structural properties for cluster long-run dynamics: Evidence from collaborative R\&D networks in the European mobile phone industry. Industrial and Corporate Change, 25(2), 261-282. doi:10.1093/icc/dtv032

Crocker, D. (1993). Making standards the IETF way. StandardView, 1(1), 48-56. doi:10.1145/174683.174689

David, P. A. (1985). Clio and the Economy of QWERTY. The American Economic Review, 75(2), 332-337.

David, P. A., \& Greenstein, S. (1990). The Economics of Compatibility Standards: An Introduction to recent research. Economics of Innovation and New Technology, 1(1-2), 3-41. doi:10.1080/10438599000000002

Delcamp, H. R., \& Leiponen, A. E. (2014). Innovating Standards Through Informal Consortia: The Case of Wireless Telecommunications. International Journal of Industrial Organization, 36, 36-47. doi:10.1016/j. ijindorg.2013.07.004

Di Minin, A., \& Bianchi, M. (2011). Safe nests in global nets: Internationalization and appropriability of R\&D in wireless telecom. Journal of International Business Studies, 42(7), 910-934. doi:10.1057/jibs.2011.16

Economides, N. (1996). Network Externalities, Complementarities and Invitations to Enter. European Journal of Political Economy, 12(2), 211-233. doi:10.1016/0176-2680(95)00014-3

Ende van den, J., Kaa van de, G., Uijl den, S., \& Vries de, H. J. (2012). The paradox of standard flexibility: The effects of co-evolution between standard and interorganizational network. Organization Studies, 33(5-6), 705-736. Doi:<ALIGNMENT.qj></ALIGNMENT>10.1177/0170840612443625 
Farrell, J., \& Klemperer, P. (2007). Chapter 31 Coordination and Lock-In: Competition with Switching Costs and Network Effects. In Handbook of Industrial Organization. Doi:<ALIGNMENT.qj></ALIGNMENT>10.1016/ S1573-448X(06)03031-7

Fransman, M. (2010). The New ICT Ecosystem: Implications for Policy and Regulation. Cambridge: Cambridge University Press. doi:10.1017/CBO9780511676130

Funk, J. L., \& Methe, D. T. (2001). Market- and committee-based mechanisms in the creation and diffusion of global industry standards: The case of mobile communication. Research Policy, 30(4), 589-610. doi:10.1016/ S0048-7333(00)00095-0

Gandal, N., Salant, D., \& Waverman, L. (2003). Standards in wireless telephone networks. Telecommunications Policy, 27(5-6), 325-332. doi:10.1016/S0308-5961(03)00026-0

Garas, A., Tomasello, M. V., \& Schweitzer, F. (2014). Selection rules in alliance formation: strategic decisions or abundance of choice? Retrieved from http://arxiv.org/abs/1403.3298

Gaynor, M., \& Bradner, S. (2001). The real options approach to standardization. Proceedings of the 34th annual Hawaii International Conference On System Sciences. http://doi.org/ doi:10.1109/HICSS.2001.926526

Gençer, M. (2007). Structure and collaborative aspects of internet standards. Proceedings of the IEEE Symposium on Computers and Communications (pp. 733-7390. http://doi.org/ doi:10.1109/ISCC.2007.4381499

Gençer, M. (2012). The evolution of IETF standards and their production. International Journal of IT Standards and Standardization Research, 10(1), 17-33. doi:10.4018/jitsr.2012010102

Granovetter, M. S. (1973). The Strength of Weak Ties. American Journal of Sociology, 78(6), 1360-1380. doi:10.1086/225469

Grøtnes, E. (2008). Strategies for Influencing the Standardization Process: Examples from Within. Proceedings of the16th European Conference on Information Systems. Association for Information Systems.

Gunasekaran, V., \& Harmantzis, F. C. (2008). Towards a Wi-Fi ecosystem: Technology integration and emerging service models. Telecommunications Policy, 32(3-4), 163-181. doi:10.1016/j.telpol.2008.01.002

Hanneman, R. A., \& Riddle, M. (2005). Introduction to social network methods. Riverside, CA: University of California. Retrieved fromhttp://faculty.ucr.edu/ hanneman/

Haug, T. (2002). A commentary on standardization practices: Lessons from the NMT and GSM mobile telephone standards histories. Telecommunications Policy, 26(3-4), 101-107. doi:10.1016/S0308-5961(02)00003-4

Hawkins, R., \& Ballon, P. (2007). When standards become business models: Reinterpreting failure in the standardization paradigm. Info, 9(5), 20-30. doi:10.1108/14636690710816426

Hillebrand, F. (2013). The creation of standards for global mobile communication: GSM and UMTS standardization from 1982 to 2000. IEEE Wireless Communications, 20(5), 24-33. doi:10.1109/MWC.2013.6664470

Isaak, J. (2006). The Role of Individuals and Social Capital in POSIX Standardization. International Journal of IT Standards and Standardization Research, 4(1), 1-23. doi:10.4018/jitsr.2006010101

Jakobs, K. (2003). Information technology standards, standards setting and standards research. Proceedings of the Cotswolds Conference, Stanhope Center for Communication Policy Research (pp. 8-9).

Jakobs, K. (2008). ICT standardisation - co-ordinating the diversity. International Telecommunication Union. Proceedings of the 1st ITU-T Kaleidoscope Academic Conference, Innovations in NGN. Doi:<ALIGNMENT. qj $></$ ALIGNMENT>10.1109/KINGN.2008.4542257

Jakobs, K., Procter, R., \& Williams, R. (2001). The Making of Standards : Looking Inside the Work Groups. IEEE Communications Magazine, 39(4), 102-107. doi:10.1109/35.917511

Kane, G. C. (2009). It's a Network, Not an Encyclopedia : A Social Network Perspective on Wikipedia Collaboration. Academy of Management Journal. doi:10.5465/AMBPP.2009.44243222

Katusic, D., Weber, M., Bojic, I., Jezic, G., \& Kusek, M. (2012). Market, standardization, and regulation development in Machine-to-Machine communications. Proceedings of the20th International Conference on Software, Telecommunications and Computer Networks (SoftCOM) (pp. 1-7). 
Katz, M. L., \& Shapiro, C. (1985). Network Externalities, Competition, and Compatibility. The American Economic Review, 75(3), 424-440.

Krämer, N. C., Rösner, L., Eimler, S., Winter, S., \& Neubaum, G. (2014). Let the Weakest Link Go! Empirical Explorations on the Relative Importance of Weak and Strong Ties on Social Networking Sites. Societies, 4(4), 785-809. doi:10.3390/soc4040785

Kwak, J. Y., Lee, H., \& Chung, D. B. (2012). The evolution of alliance structure in Chinas mobile telecommunication industry and implications for international standardization. Telecommunications Policy, 36(10-11), 966-976. doi:10.1016/j.telpol.2012.07.017

Lehr, W. H., \& McKnight, L. W. (2003). Wireless Internet access: 3G vs. WiFi? Telecommunications Policy, 27(5-6), 351-370. doi:10.1016/S0308-5961(03)00004-1

Leiner, B. M., Cerf, V. G., Clark, D. D., Kahn, R. E., Kleinrock, L., Lynch, D. C., \& Wolf, S. et al. (2009). A Brief History of the Internet. Computer Communication Review, 39(5), 22-31. doi:10.1145/1629607.1629613

Leiponen, A. E. (2008). Competing Through Cooperation: The Organization of Standard Setting in Wireless Telecommunications. Management Science, 54(11), 1904-1919. doi:10.1287/mnsc.1080.0912

Lemstra, W., \& Hayes, V. (2009). License-exempt: Wi-Fi complement to 3G. Telematics and Informatics, 26(3), 227-239. doi:10.1016/j.tele.2008.11.003

Lemstra, W., Hayes, V., \& Groenewegen, J. (2011). The Innovation Journey of Wi-Fi. The Innovation Journey of Wi-Fi: The Road to Global Success. Cambridge: University Press; doi:10.1017/CBO9780511666995

Lerner, J., \& Tirole, J. (2006). A Model of Forum Shopping, with Special Reference to Standard Setting Organizations. The American Economic Review, 96(4), 1091-1113. doi:10.1257/aer.96.4.1091

Lyytinen, K., \& King, J. L. (2002). Around the cradle of the wireless revolution: The emergence and evolution of cellular telephony. Telecommunications Policy, 26(3-4), 97-100. doi:10.1016/S0308-5961(02)00002-2

Majchrzak, A., Jarvenpaa, S. L., \& Bagherzadeh, M. (2015). A Review of Interorganizational Collaboration Dynamics. Journal of Management, 41(5), 1338-1360. doi:10.1177/0149206314563399

Majchrzak, A., Jarvenpaa, S. L., \& Bagherzadeh, M. (2015). A Review of Interorganizational Collaboration Dynamics. Journal of Management, 41(5), 1338-1360. doi:10.1177/0149206314563399

Martinez-Romo, J., Robles, G., Gonzalez-Barahona, J. M., \& Ortuño-Perez, M. (2008). Using Social Network Analysis Techniques to Study Collaboration between a FLOSS Community and a Company. IFIP International Federation for Information Processing, 275, 171-186. doi:10.1007/978-0-387-09684-1_14

Newman, M. E. J. (2001). The structure of scientific collaboration networks. Proceedings of the National Academy of Sciences of the United States of America, 98(2), 404-409. doi:10.1073/pnas.98.2.404 PMID:11149952

Noldus, R., \& Van Mieghem, P. (2014). Assortativity in complex networks. Journal of Complex Networks, 3(4), 507-542. doi:10.1093/comnet/cnv005

Pohlmann, T. (2014). The Evolution of ICT Standards Consortia. The DigiWorld Economic Journal, (95), 17-40.

Prim, R. C. (1957). Shortest Connection Networks And Some Generalizations. The Bell System Technical Journal, 36(6), 1389-1401. doi:10.1002/j.1538-7305.1957.tb01515.x

Rogers, E. M. (1995). Diffusion of innovations. New York, NY: Free Press.

Shin, D. H., Kim, H., \& Hwang, J. (2015). Standardization revisited: A critical literature review on standards and innovation. Computer Standards \& Interfaces, 38, 152-157. doi:10.1016/j.csi.2014.09.002

Simcoe, T. (2012). Standard setting committees: Consensus governance for shared technology platforms. The American Economic Review, 102(1), 305-336. doi:10.1257/aer.102.1.305

Simcoe, T. (2015). Modularity and the evolution of the Internet. In Economical Analysis of the Digital Economy (pp. 21-54). Chicago: University of Chicago Press.

Sterman, J. D. (2002). All models are wrong: Reflections on becoming a systems scientist. System Dynamics Review, 18(4), 501-531. doi:10.1002/sdr.261 
Strogatz, S. H. (2001). Exploring complex networks. Nature, 410(6825), 268-276. doi:10.1038/35065725 PMID:11258382

Swann, P. G. M. (2010). The Economics of Standardization: An Update.

Tilson, D., \& Lyytinen, K. (2006). The 3G transition: Changes in the US wireless industry. Telecommunications Policy, 30(10-11), 569-586. doi:10.1016/j.telpol.2006.09.002

Toppila, A., Liesiö, J., \& Salo, A. (2011). A Resource Allocation Model for R\&D Investments: A Case Study in Telecommunication Standardization. In A. Salo, J. Keisler, \& A. Morton (Eds.), Portfolio Decision Analysis: Improved methods for resource allocation (pp. 241-258). New York, NY: Springer New York; doi:10.1007/9781-4419-9943-6_11

Trestian, R., Ormond, O., \& Muntean, G. (2012). Game Theory - Based Network Selection : Solutions and Challenges. Communications Survey \& Tutorials, 14(4), 1212-1231. doi:10.1109/SURV.2012.010912.00081

van de Kaa, G., \& de Bruijn, H. (2015). Platforms and incentives for consensus building on complex ICT systems: The development of WiFi. Telecommunications Policy, 39(7), 580-589. doi:10.1016/j.telpol.2014.12.012

van de Kaa, G., \& de Vries, H. J. (2015). Factors for winning format battles: A comparative case study. Technological Forecasting and Social Change, 91, 222-235. doi:10.1016/j.techfore.2014.02.019

van de Kaa, G., van den Ende, J., de Vries, H. J., \& van Heck, E. (2011). Factors for winning interface format battles: A review and synthesis of the literature. Technological Forecasting and Social Change, 78(8), 1397-1411. doi:10.1016/j.techfore.2011.03.011

Vasudeva, G., Alexander, E. A., \& Jones, S. L. (2015). Institutional Logics and Interorganizational Learning in Technological Arenas: Evidence from Standard-Setting Organizations in the Mobile Handset Industry. Organization Science, 26(3), 830-846. doi:10.1287/orsc.2014.0940

Volpentesta, A. P., \& Felicetti, A. M. (2010). Eigenvector centrality based on shared research topics in a scientific community. In IFIP Advances in Information and Communication Technology (pp. 626-633). Doi:<ALIGNMENT.qj></ALIGNMENT>10.1007/978-3-642-15961-9_75

Von Bertalanffy, L. (1972). The history and status of general systems theory. Academy of Management Journal, 15(4), 407-426. doi:10.2307/255139

Wasserman, S., \& Faust, K. (1994). Social network analysis: Methods and applications. Cambridge: University Press. doi:10.1017/CBO9780511815478

Watts, D. J., \& Strogatz, S. (1998). Collective dynamics of small-world networks. Nature, 393(6684), 440-442. doi:10.1038/30918 PMID:9623998

Weiss, M. B. H., \& Sirbu, M. (1990). Technological choice in voluntary standards committees: An empirical analysis. Economics of Innovation and New Technology, 1(1-2), 111-133. doi:10.1080/10438599000000007

\section{ENDNOTES}

http://www.etsi.org/about/what-we-are

https://www.bluetooth.com/media/our-history

In this paper, abbreviation "SSO" refers to any kind of standard-setting group or activity including SSOs developing, defining or mandating standards.

4 In this paper, "Collaboration" refers to "Cooperative and "Coopetitive" behaviors while "Competitive" refers to stand-alone approach. Collaborating contributor aligns with the connected node of a social network while Competing-only contributors refers to disconnected nodes. Request For Comments, referring to the documents used in IETF. http://www.darpa.mil/about-us/darpa-history-and-timeline http://home.cern/about https://www.ieee.org/about/ieee_history.html http://www.internetsociety.org/history http://www.internetworldstats.com/emarketing.htm 
https://www.w3.org/History.html

IEEE-SA claims 183 corporate members

Wi-Fi Alliance is a founded in 1999 to drive the global acceptance and adoption of the IEEE 802.11 standards. Wi-Fi is the trademark of the WFA. http://www.wi-fi.org/ https://www.ansi.org/about_ansi/overview/overview.aspx?menuid=1 https://share.ansi.org/Shared\%20Documents/Standards\%20Activities/American\%20National\%20 Standards/ANSI\%20Accredited\%20Standards\%20Developers/AUG2016ASD.pdf http://www.cept.org/ http://www.atis.org/01_about/our_work.asp http://www.ccsa.org.cn/english/about.php http://www.3gpp.org/ftp/tsg_sa/TSG_SA/TSGS_17/Docs/PDF/SP-020418.pdf http://www.sipforum.org/content/view/13/34/

Marketing name for IEEE 802.16 based wide area wireless technology.

It is for further study to evaluate different structures of the elementary networks by assigning different weights to the different links based on the further interviews of the standardization experts.

See appendix 1 for more details

The hyperlinks are directed while the standardization collaboration connections in this research are undirected.

Empirical data for each SSO is available differently. Assortativity is visible for all years where sensible amount of data is available noting that the number of documents outside of the research focus area 2003 -2008 limited.

The IEEE $802.11 \mathrm{n}$ standard was technically ready in 2007 but it took two more years to achieve the final acceptance of the work.

There is a number of 3GPP standards developed in the release independent fashion.

The documents contributed in the WFA are not available for this research and therefore the impact of WFA is not taken into account quantitatively in this analysis.

http://www.globalcertificationforum.org/images/downloads/GCF-WP-GCF_Certification_June2014.pdf 


\section{APPENDIX}

\section{A Brief Introduction to Social Network Analysis}

Social networks analysis (SNA) and the tools used in this study have been broadly adopted (Wasserman $\&$ Faust, 1994). This appendix provides only a brief introduction of these methods. Widely used open source software R Studio with specific "igraph" library was used to compute the algorithms and functions.

A common way to model social networks is to define the relation $\mathrm{k}$ (link, edge) between the network actors $n_{i}$ and $n_{j}$ (node, vertex) in the form of an Adjacency matrix A. The elements of the matrix A are defined as:

$$
a_{i, j}=\left\{\begin{array}{l}
w ; \quad \text { when there is } w \text { links between node } n_{i} \text { and } n_{j} \\
0 ; \quad \text { when there is no link }
\end{array}\right.
$$

In this research, link weights reflect activity or intensity of the collaboration. Quality of the networking driven collaboration is studied based on network topology where multiple links are ignored and hence the weights are all equal to 1 . The $\mathrm{N}$ nodes in the network yield $\mathrm{N} x \mathrm{~N}$ dimensional adjacency matrix A. Therefore, $\mathrm{N}$ denotes also Neighborhood.

Elements on the diagonal of the matrix A (for $a_{i, j}$ where $\mathrm{i}=\mathrm{j}$ ) are normally ignored when collaboration in the networks are studies. The diagonal reflects "self-collaboration" or in practice a competitive action. For statistical analysis, the values on diagonal are included but for SNA the values are ignored.

Transitivity is a simple but powerful property of social networks. Parallel definition for transitivity is Clustering Coefficient. In this study, the term transitivity is used. High transitivity is an early indication of a social rather than a random network. Formally, the transitivity $\mathrm{T}$ is the ratio of closed triplets of nodes and the number of all connected triplets in the network. Transitivity is a measure if a friend of my friend is also my friend (Wasserman \& Faust, 1994).

Global Transitivity $\mathrm{T}$ over the whole network follows the Equation 2:

$$
T=\frac{\text { Number of transitive triplets }}{\text { Number of potential transitive triplets }}
$$

Separately Average Local Transitivity $\bar{T}$ is an average of local transitivity values of all nodes:

$$
\bar{T}=\frac{1}{N} \sum_{i=1}^{N} T_{i}
$$

Disconnected nodes do not count because the interest to consider potential friendships of nonexisting friends is obviously low. Furthermore, the friendship is taken into account in an equal manner independently of how active the friendship is, i.e. links are considered unweighted.

Low Average path length L in highly clustered networks (high transitivity) is an indicator of so-called small world network. The average path length is a sum of all the measured shortest path lengths $d_{i j}$ averaged over all pairs of nodes: 


$$
L=\frac{1}{N(N-1)} \sum_{i, j=1}^{N}\left(d_{i j}\right) ; i \neq j
$$

Degree of a network node indicates the number of the links that connect the node to the other nodes in the network. Weighted degree takes into account also the parallel links between the two nodes. Degree does not include the elements on the diagonal of adjacency matrix A. The Degree of a node is defined as:

$$
D_{i}=\sum_{j=1}^{N} a_{i j} ; i \neq j
$$

and for weighted links:

$$
D_{i}^{w}=\sum_{j=1}^{N} w_{j} a_{i j} ; i \neq j
$$

Node degree is a simple measure of importance of the node in a network. Therefore, node degree equal to Degree Centrality of the node. For instance, analysis on the Wikipedia has found that the quality of articles is depending on the position of the article and the authors within both local and global networks. Weighted degree centrality captures the activity centrality of a node in the local network by calculating the number and strength of the relationships of which the node is involved (Kane, 2009). This study looks at the weighted degree centrality ranking of the nodes in order to sort the standardization contributors into the order of their collaborative activity. The steeper slope of the curve is an indication of shorter tail of the ranking and higher degree centralization of the network (Crespo et al., 2014).

Eigenvector centrality utilizes the idea that the role of a node is more central if it is connected to other nodes that are themselves central as well. While degree centrality measures the local connections of a node, eigenvector centrality is a total assessment of collaboration (influence) over all the connections in the network. Eigenvector centrality captures the centrality of a node globally by weighting the value of a node's direct ties as a function of all the relationships those nodes are involved (Kane, 2009). This way, social aspects of Wikipedia have been found to be indispensable for understanding the collaborative processes. Eigenvector centrality relates closely to Page Rank index of directed hyperlinks in the World Wide Web (WWW). Page Rank is a key metric of e.g. state of the art search engines. Eigenvector centrality has been used to analyze for instance collaboration in scientific community (Volpentesta \& Felicetti, 2010). Eigenvector centrality is defined as values of the first eigenvector (i.e. corresponding to the largest eigenvalue) of the adjacency matrix A. Each node $a_{n}$ will have its centrality score defined as:

$$
s_{i}=1 / \lambda \sum_{j=1}^{N} a_{i, j} s_{j}
$$

which can be rewritten as the eigenvector equation: 
where $\lambda$ represents the eigenvalues of the adjacency matrix $A$ and $\lambda_{1}$ is the largest eigenvalue corresponding the eigenvector used to define the eigenvector centrality. The link weights are not used.

Assortativity is a measure of degree correlation where a statistical correlation between the degrees of connected nodes is calculated. High correlation between connected nodes and their degrees in the network is considered assortative. If the correlation is negative, the network is considered disassortative (Noldus \& Mieghem, 2014).

Maximum spanning tree is a qualitative view to the collaboration network. Maximum spanning tree is an inversion of minimum spanning tree, which connects all the nodes of a network with minimum weight. Early idea of searching such shortest connections emerged from the need to optimize connections in large telephone networks (Prim, 1957). Computation of the maximum spanning tree re-use the minimum spanning tree algorithms with negative link weights.

SNA tools listed above take into account only the connected nodes (degree $D_{i} \geq 1$ ). Significant amount of standardization contributions is provided by single source, standalone contributors (degree $D_{i}=0$ ). Therefore, the role of such formally non-collaborative or competitive contributions require at least some attention. Another option would be to consider the stand-alone contributions similar to very weak ties. The role of weak ties have been studied for several decades (Granovetter, 1973) with also controversial perspectives (Krämer, Rösner, Eimler, Winter \& Neubaum, 2014). In this research, these "invisible" very weak ties are ignored in the quantitative SNA but are taken into account semiquantitatively in maximum spanning tree pictures and in qualitative interviews.

Furthermore, a quantitative view is provided by a simple statistical analysis of the ratios between competitive, collaborative and coopetitive contributors in each SSO (Majchrzak et al., 2015).

Timo Ali-Vehmas, Nokia Fellow, is Head of Ecosystems Research, Nokia Technologies. Timo Ali-Vehmas was nominated in 2015 to lead the emerging ecosystem research in Nokia Technologies. The knowledge and leadership related to ecosystems is needed to complement the competences and capabilities in technologies. The ICT driven ecosystems based on the collaboration within and between the industries is one of the drivers of next generation of systems and services. Before the current role since 2005 he was in charge of Nokia's standardization activities as VP, Compatibility and Industry Collaboration, including Nokia's contributions in forums like 3GPP, ETSI, CCSA, IEEE, ITU, IETF, W3C, OMA, DLNA, WFA, BT and many others. He has been working in Nokia since 1980 in a number of different areas, including R\&D manager of the first Nokia GSM mobile phone, Head of Radio Communication Laboratory in Nokia Research Centre and VP, WCDMA Product Programs in Nokia Mobile Phones. Since 2012 Timo Ali-Vehmas has served as the Chairman of the Board of Nokia Foundation. Since 2008 he has been member also the Board of Walter Ahlström Foundation. Timo Ali-Vehmas graduated at Helsinki University of Technology 1982. 